
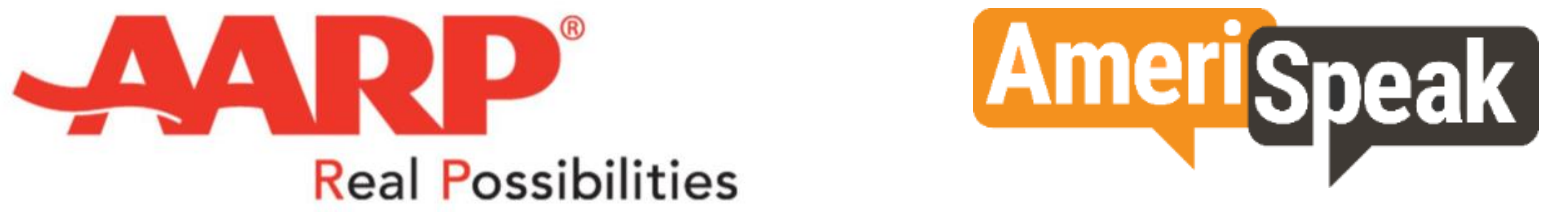

\title{
50+ Coronavirus Tracker: Wave 5 Oversample
}

\section{Conducted by NORC at the University of Chicago for AARP}

Interviews: 5/29/2020-06/09/2020

1,798 adults

Margin of error: 3.37 percentage points at the 95\% confidence level among all adults

NOTE: All results show percentages among all respondents, unless otherwise labeled.

Percentages may not always sum to $100 \%$.

"*" indicates less than $0.5 \%$ "-" indicates $0 \%$

https://doi.org/10.26419/res.00385.009 
Q1. How concerned would you say you are about the coronavirus?

\begin{tabular}{|l|c|c|c|c|c|}
\hline $\begin{array}{l}\text { NORC } \\
\text { 05/29-06/09/2020 }\end{array}$ & Total & White NH & $\begin{array}{c}\text { African } \\
\text { American } \\
\text { NH }\end{array}$ & Hispanic & Other NH \\
\hline Very concerned & 53 & 46 & 75 & 47 & 53 \\
\hline Somewhat concerned & 33 & 41 & 21 & 30 & 26 \\
\hline Not very concerned & 10 & 11 & 1 & 14 & 17 \\
\hline Not at all concerned & 4 & 3 & 2 & 7 & 4 \\
\hline $\begin{array}{l}\text { DON'T KNOW/ SKIPPED } \\
\text { ON WEB/ REFUSED }\end{array}$ & 1 & - & 1 & 3 & - \\
\hline
\end{tabular}

Q2a. Have you ever had coronavirus (suspected or diagnosed) at any point since December of 2019?

\begin{tabular}{|l|c|c|c|c|c|}
\hline $\begin{array}{l}\text { NORC } \\
05 / 29-06 / 09 / 2020\end{array}$ & Total & White NH & $\begin{array}{c}\text { African } \\
\text { American NH }\end{array}$ & Hispanic & Other NH \\
\hline Yes & 6 & 6 & 4 & 5 & 13 \\
\hline No & 94 & 93 & 95 & 95 & 87 \\
\hline $\begin{array}{l}\text { DON'T KNOW/ SKIPPED } \\
\text { ON WEB/ REFUSED }\end{array}$ & $*$ & 1 & 1 & - & - \\
\hline
\end{tabular}

Q2b. Have you been diagnosed with coronavirus by a medical professional? Please include a doctor diagnosis with or without a coronavirus test, or if a doctor or medical professional has recommended you stay home due to symptoms matching coronavirus.

\begin{tabular}{|l|c|c|c|c|c|}
\hline $\begin{array}{l}\text { NORC } \\
\text { 05/29-06/09/2020 }\end{array}$ & Total & White NH & $\begin{array}{c}\text { African } \\
\text { American } \\
\mathrm{NH}\end{array}$ & Hispanic & Other NH \\
\hline Yes & 1 & 1 & 3 & 1 & - \\
\hline No & 98 & 99 & 97 & 99 & 100 \\
\hline $\begin{array}{l}\text { DON'T KNOW/ SKIPPED } \\
\text { ON WEB/ REFUSED }\end{array}$ & $*$ & $*$ & $*$ & - & - \\
\hline
\end{tabular}


AARP 50+ Coronavirus Tracker: Wave 5 Oversample

Has been diagnosed with coronavirus

Q2c. Did you have a positive coronavirus test result?

\begin{tabular}{|l|c|c|c|c|c|}
\hline $\begin{array}{l}\text { NORC } \\
\text { 05/29-06/09/2020 }\end{array}$ & Total & White NH & $\begin{array}{c}\text { African } \\
\text { American } \\
\text { NH }\end{array}$ & Hispanic & $\begin{array}{c}\text { Other } \\
\text { NH }\end{array}$ \\
\hline Yes & $*$ & $*$ & $*$ & $*$ & - \\
\hline No & $*$ & $*$ & $*$ & $*$ & - \\
\hline Test still pending & $*$ & $*$ & $*$ & $*$ & - \\
\hline $\begin{array}{l}\text { DON'T KNOW/ SKIPPED ON WEB/ } \\
\text { REFUSED }\end{array}$ & $*$ & $*$ & $*$ & $*$ & - \\
\hline
\end{tabular}

Note: Base sizes too small to report percentages.

Q3. How many people do you personally know who have been infected by COVID (or "coronavirus")?

\begin{tabular}{|l|c|c|c|c|c|}
\hline $\begin{array}{l}\text { NORC } \\
\text { 05/29-06/02/2020 }\end{array}$ & Total & $\begin{array}{c}\text { White } \\
\text { NH }\end{array}$ & $\begin{array}{c}\text { African } \\
\text { American } \\
\text { NH }\end{array}$ & Hispanic & $\begin{array}{c}\text { Other } \\
\text { NH }\end{array}$ \\
\hline 1 & 12 & 11 & 14 & 13 & 7 \\
\hline 2 & 9 & 8 & 11 & 8 & 6 \\
\hline 3 & 5 & 5 & 9 & 4 & 2 \\
\hline 4 & 4 & 4 & 5 & 5 & 2 \\
\hline 5 & 3 & 3 & 4 & 2 & - \\
\hline 6 & 2 & 1 & 3 & 1 & 5 \\
\hline 7 & $*$ & $*$ & 1 & $*$ & - \\
\hline 8 & $*$ & - & 1 & 1 & - \\
\hline 9 & $*$ & $*$ & $*$ & 1 & - \\
\hline 10 & 1 & 1 & 2 & $*$ & 3 \\
\hline 11 & $*$ & - & $*$ & - & - \\
\hline 12 & $*$ & $*$ & $*$ & - & - \\
\hline 13 & $*$ & $*$ & $*$ & - & - \\
\hline 15 & 1 & $*$ & 1 & 1 & 2 \\
\hline 18 & $*$ & - & - & $*$ & - \\
\hline 20 & $*$ & - & - & $*$ & - \\
\hline 25 & $*$ & $*$ & $*$ & - & - \\
\hline 30 & $*$ & - & $*$ & $*$ & - \\
\hline 50 & $*$ & - & $*$ & - & - \\
\hline 99 & $*$ & - & 1 & - & - \\
\hline I do not personally know anyone \\
infected by COVID & 62 & 67 & 47 & 63 & 72 \\
\hline DON'T KNOW/ SKIPPED ON WEB/ & $* 1,798$ & $N=906$ & $N=401$ & $N=444$ & $N=47$ \\
\hline REFUSED & & 1 & 1 & 1 & 1 \\
\hline
\end{tabular}


If knows someone personally infected with COVID

Q4. What was the outcome for the people you personally know who have been infected with COVID?

Please select all that apply.

\begin{tabular}{|l|c|c|c|c|c|}
\hline $\begin{array}{l}\text { NORC } \\
\text { 05/29-06/02/2020 }\end{array}$ & Total & White NH & $\begin{array}{c}\text { African } \\
\text { American } \\
\mathrm{NH}\end{array}$ & Hispanic & $\begin{array}{c}\text { Other } \\
\mathrm{NH}\end{array}$ \\
\hline Recovered from the infection & 72 & 81 & 67 & 62 & $*$ \\
\hline Still sick or recovering & 22 & 23 & 23 & 17 & $*$ \\
\hline Died from the infection & 36 & 25 & 44 & 48 & $*$ \\
\hline Unknown & 5 & 3 & 7 & 5 & $*$ \\
\hline $\begin{array}{l}\text { DON'T KNOW/ SKIPPED ON WEB/ } \\
\text { REFUSED }\end{array}$ & 1 & - & 2 & 1 & $*$ \\
\hline
\end{tabular}

Note: Base sizes too small to report percentages for Other NH.

Q5. Some people have found the guidelines for preventing the spread of the coronavirus to not always be practical or easy to follow in all situations. How about for you? How often are you personally following guidelines such as social distancing, washing hands frequently, restricting travel and shopping trips, etc.)?

\begin{tabular}{|l|c|c|c|c|c|}
\hline $\begin{array}{l}\text { NORC } \\
\text { 05/29-06/09/2020 }\end{array}$ & Total & White NH & $\begin{array}{c}\text { African } \\
\text { American } \\
\mathrm{NH}\end{array}$ & Hispanic & $\begin{array}{c}\text { Other } \\
\mathrm{NH}\end{array}$ \\
\hline Following all the time & 52 & 49 & 66 & 49 & 44 \\
\hline Following most of the time & 36 & 38 & 29 & 36 & 38 \\
\hline Following some of the time & 9 & 11 & 3 & 10 & 13 \\
\hline $\begin{array}{l}\text { Following very little or none of the } \\
\text { time }\end{array}$ & 3 & 2 & 1 & 5 & 5 \\
\hline $\begin{array}{l}\text { DON'T KNOW/ SKIPPED ON WEB/ } \\
\text { REFUSED }\end{array}$ & $*$ & $*$ & 1 & - & - \\
\hline
\end{tabular}


Q6. How worried, if at all, are you that...

[ITEMS RANDOMIZED]

\begin{tabular}{|c|c|c|c|c|c|}
\hline $\begin{array}{l}\text { Total } \\
05 / 29-06 / 09 / 2020\end{array}$ & $\begin{array}{c}\text { Very } \\
\text { worried }\end{array}$ & $\begin{array}{l}\text { Somewhat } \\
\text { worried }\end{array}$ & $\begin{array}{l}\text { Not very } \\
\text { worried }\end{array}$ & $\begin{array}{l}\text { Not at all } \\
\text { worried }\end{array}$ & $\begin{array}{l}\text { DON'T } \\
\text { KNOW/ } \\
\text { SKIPPED } \\
\text { ON WEB/ } \\
\text { REFUSED }\end{array}$ \\
\hline $\begin{array}{l}\text { You or someone in your family will get sick from } \\
\text { the coronavirus }\end{array}$ & 27 & 33 & 23 & 16 & $*$ \\
\hline $\begin{array}{l}\text { You or someone in your family will die from the } \\
\text { coronavirus }\end{array}$ & 21 & 29 & 28 & 22 & 1 \\
\hline $\begin{array}{l}\text { Your investments such as retirement or college } \\
\text { savings will be negatively impacted by coronavirus }\end{array}$ & 17 & 27 & 22 & 34 & 1 \\
\hline $\begin{array}{l}\text { You will not be able to afford testing or treatment } \\
\text { for coronavirus if you need it }\end{array}$ & 12 & 16 & 27 & 45 & 1 \\
\hline $\begin{array}{l}\text { You will not be able to care for a family member or } \\
\text { friend }\end{array}$ & 15 & 26 & 26 & 33 & $*$ \\
\hline $\begin{array}{l}\text { Your existing health conditions will put you at } \\
\text { greater risk of contracting the coronavirus }\end{array}$ & 19 & 29 & 26 & 26 & $*$ \\
\hline $\begin{array}{l}\text { You will be the victim of a scam about the } \\
\text { coronavirus }\end{array}$ & 8 & 15 & 29 & 48 & 1 \\
\hline You will feel more isolated or lonely & 9 & 24 & 30 & 36 & 1 \\
\hline You will have to postpone your retirement plans & 10 & 16 & 19 & 54 & 1 \\
\hline You will have to help neighbors financially & 5 & 13 & 28 & 52 & 2 \\
\hline You will have to help family financially & 10 & 22 & 32 & 36 & 1 \\
\hline $\begin{array}{l}\text { You will not be able to get the groceries or } \\
\text { medications you need }\end{array}$ & 10 & 24 & 31 & 34 & 1 \\
\hline $\begin{array}{l}\text { You will not be able to vote in the Presidential } \\
\text { election in November }\end{array}$ & 11 & 16 & 23 & 48 & 1 \\
\hline $\begin{array}{l}\text { You are concerned your Social Security benefits } \\
\text { could be reduced }\end{array}$ & 19 & 26 & 23 & 31 & 1 \\
\hline You will lose savings & 15 & 26 & 27 & 32 & 1 \\
\hline You will lose investments & 16 & 26 & 21 & 36 & 1 \\
\hline You will have to delay bill payments & 11 & 19 & 27 & 42 & 1 \\
\hline $\begin{array}{l}\text { You might have to use retirement savings to pay } \\
\text { for necessary expenses }\end{array}$ & 12 & 22 & 28 & 37 & 1 \\
\hline $\begin{array}{l}\text { You will have to lend money to help family or } \\
\text { friends because they were impacted financially by } \\
\text { coronavirus }\end{array}$ & 10 & 19 & 35 & 35 & 1 \\
\hline $\begin{array}{l}\text { You will lose income from "gig" and side work, like } \\
\text { Uber, Airbnb, housesitting or dog-walking }\end{array}$ & 6 & 11 & 16 & 67 & 1 \\
\hline $\begin{array}{l}\text { You will lose your job, be furloughed, or have your } \\
\text { work suspended }\end{array}$ & 10 & 13 & 17 & 59 & 1 \\
\hline
\end{tabular}

$N=1,798$ 


\begin{tabular}{|c|c|c|c|c|c|}
\hline $\begin{array}{l}\text { White NH } \\
\text { 05/29-06/09/2020 }\end{array}$ & $\begin{array}{c}\text { Very } \\
\text { worried }\end{array}$ & $\begin{array}{l}\text { Somewhat } \\
\text { worried }\end{array}$ & $\begin{array}{l}\text { Not very } \\
\text { worried }\end{array}$ & $\begin{array}{l}\text { Not at all } \\
\text { worried }\end{array}$ & $\begin{array}{l}\text { DON'T } \\
\text { KNOW/ } \\
\text { SKIPPED } \\
\text { ON WEB/ } \\
\text { REFUSED }\end{array}$ \\
\hline $\begin{array}{l}\text { You or someone in your family will get sick from } \\
\text { the coronavirus }\end{array}$ & 21 & 36 & 28 & 15 & $*$ \\
\hline $\begin{array}{l}\text { You or someone in your family will die from the } \\
\text { coronavirus }\end{array}$ & 16 & 30 & 32 & 20 & 1 \\
\hline $\begin{array}{l}\text { Your investments such as retirement or college } \\
\text { savings will be negatively impacted by coronavirus }\end{array}$ & 17 & 29 & 22 & 31 & 1 \\
\hline $\begin{array}{l}\text { You will not be able to afford testing or treatment } \\
\text { for coronavirus if you need it }\end{array}$ & 6 & 14 & 29 & 51 & 1 \\
\hline $\begin{array}{l}\text { You will not be able to care for a family member or } \\
\text { friend }\end{array}$ & 11 & 26 & 31 & 32 & $*$ \\
\hline $\begin{array}{l}\text { Your existing health conditions will put you at } \\
\text { greater risk of contracting the coronavirus }\end{array}$ & 17 & 32 & 28 & 23 & $*$ \\
\hline $\begin{array}{l}\text { You will be the victim of a scam about the } \\
\text { coronavirus }\end{array}$ & 4 & 13 & 33 & 49 & 1 \\
\hline You will feel more isolated or lonely & 7 & 27 & 33 & 33 & 1 \\
\hline You will have to postpone your retirement plans & 6 & 15 & 19 & 59 & 1 \\
\hline You will have to help neighbors financially & 4 & 9 & 31 & 56 & 1 \\
\hline You will have to help family financially & 7 & 20 & 36 & 37 & $*$ \\
\hline $\begin{array}{l}\text { You will not be able to get the groceries or } \\
\text { medications you need }\end{array}$ & 6 & 24 & 36 & 34 & 1 \\
\hline $\begin{array}{l}\text { You will not be able to vote in the Presidential } \\
\text { election in November }\end{array}$ & 9 & 17 & 23 & 51 & $*$ \\
\hline $\begin{array}{l}\text { You are concerned your Social Security benefits } \\
\text { could be reduced }\end{array}$ & 16 & 28 & 27 & 29 & 1 \\
\hline You will lose savings & 11 & 29 & 30 & 30 & 1 \\
\hline You will lose investments & 16 & 30 & 23 & 31 & 1 \\
\hline You will have to delay bill payments & 6 & 14 & 31 & 48 & 1 \\
\hline $\begin{array}{l}\text { You might have to use retirement savings to pay } \\
\text { for necessary expenses }\end{array}$ & 9 & 20 & 34 & 37 & $*$ \\
\hline $\begin{array}{l}\text { You will have to lend money to help family or } \\
\text { friends because they were impacted financially by } \\
\text { coronavirus }\end{array}$ & 6 & 17 & 39 & 38 & $*$ \\
\hline $\begin{array}{l}\text { You will lose income from "gig" and side work, like } \\
\text { Uber, Airbnb, housesitting or dog-walking }\end{array}$ & 4 & 8 & 14 & 74 & 1 \\
\hline $\begin{array}{l}\text { You will lose your job, be furloughed, or have your } \\
\text { work suspended }\end{array}$ & 7 & 13 & 17 & 62 & 1 \\
\hline
\end{tabular}




\begin{tabular}{|c|c|c|c|c|c|}
\hline $\begin{array}{l}\text { African American } \mathrm{NH} \\
05 / 29-06 / 09 / 2020\end{array}$ & $\begin{array}{c}\text { Very } \\
\text { worried }\end{array}$ & $\begin{array}{l}\text { Somewhat } \\
\text { worried }\end{array}$ & $\begin{array}{l}\text { Not very } \\
\text { worried }\end{array}$ & $\begin{array}{l}\text { Not at all } \\
\text { worried }\end{array}$ & $\begin{array}{c}\text { DON'T } \\
\text { KNOW/ } \\
\text { SKIPPED } \\
\text { ON } \\
\text { WEB/ } \\
\text { REFUSED }\end{array}$ \\
\hline $\begin{array}{l}\text { You or someone in your family will get sick from } \\
\text { the coronavirus }\end{array}$ & 33 & 32 & 16 & 18 & 1 \\
\hline $\begin{array}{l}\text { You or someone in your family will die from the } \\
\text { coronavirus }\end{array}$ & 26 & 30 & 24 & 20 & 1 \\
\hline $\begin{array}{l}\text { Your investments such as retirement or college } \\
\text { savings will be negatively impacted by coronavirus }\end{array}$ & 18 & 22 & 15 & 45 & * \\
\hline $\begin{array}{l}\text { You will not be able to afford testing or treatment } \\
\text { for coronavirus if you need it }\end{array}$ & 18 & 19 & 22 & 40 & 1 \\
\hline $\begin{array}{l}\text { You will not be able to care for a family member or } \\
\text { friend }\end{array}$ & 19 & 26 & 19 & 35 & 1 \\
\hline $\begin{array}{l}\text { Your existing health conditions will put you at } \\
\text { greater risk of contracting the coronavirus }\end{array}$ & 20 & 31 & 21 & 28 & 1 \\
\hline $\begin{array}{l}\text { You will be the victim of a scam about the } \\
\text { coronavirus }\end{array}$ & 9 & 20 & 23 & 46 & 2 \\
\hline You will feel more isolated or lonely & 9 & 20 & 27 & 44 & $*$ \\
\hline You will have to postpone your retirement plans & 15 & 15 & 18 & 50 & 1 \\
\hline You will have to help neighbors financially & 8 & 16 & 28 & 48 & 1 \\
\hline You will have to help family financially & 12 & 25 & 27 & 36 & 1 \\
\hline $\begin{array}{l}\text { You will not be able to get the groceries or } \\
\text { medications you need }\end{array}$ & 12 & 25 & 26 & 37 & $*$ \\
\hline $\begin{array}{l}\text { You will not be able to vote in the Presidential } \\
\text { election in November }\end{array}$ & 14 & 18 & 21 & 45 & 1 \\
\hline $\begin{array}{l}\text { You are concerned your Social Security benefits } \\
\text { could be reduced }\end{array}$ & 20 & 24 & 19 & 37 & 1 \\
\hline You will lose savings & 21 & 24 & 19 & 36 & 1 \\
\hline You will lose investments & 18 & 18 & 18 & 46 & $*$ \\
\hline You will have to delay bill payments & 16 & 27 & 22 & 35 & 1 \\
\hline $\begin{array}{l}\text { You might have to use retirement savings to pay } \\
\text { for necessary expenses }\end{array}$ & 16 & 23 & 19 & 42 & 1 \\
\hline $\begin{array}{l}\text { You will have to lend money to help family or } \\
\text { friends because they were impacted financially by } \\
\text { coronavirus }\end{array}$ & 11 & 25 & 30 & 33 & 1 \\
\hline $\begin{array}{l}\text { You will lose income from "gig" and side work, like } \\
\text { Uber, Airbnb, housesitting or dog-walking }\end{array}$ & 7 & 14 & 21 & 56 & 2 \\
\hline $\begin{array}{l}\text { You will lose your job, be furloughed, or have your } \\
\text { work suspended }\end{array}$ & 12 & 15 & 15 & 58 & 1 \\
\hline
\end{tabular}




\begin{tabular}{|c|c|c|c|c|c|}
\hline $\begin{array}{l}\text { Hispanic } \\
05 / 29-06 / 09 / 2020\end{array}$ & $\begin{array}{c}\text { Very } \\
\text { worried }\end{array}$ & $\begin{array}{l}\text { Somewhat } \\
\text { worried }\end{array}$ & $\begin{array}{l}\text { Not very } \\
\text { worried }\end{array}$ & $\begin{array}{c}\text { Not at all } \\
\text { worried }\end{array}$ & $\begin{array}{c}\text { DON'T } \\
\text { KNOW/ } \\
\text { SKIPPED } \\
\text { ON } \\
\text { WEB/ } \\
\text { REFUSED }\end{array}$ \\
\hline $\begin{array}{l}\text { You or someone in your family will get sick from } \\
\text { the coronavirus }\end{array}$ & 33 & 31 & 22 & 15 & $*$ \\
\hline $\begin{array}{l}\text { You or someone in your family will die from the } \\
\text { coronavirus }\end{array}$ & 28 & 26 & 21 & 25 & 1 \\
\hline $\begin{array}{l}\text { Your investments such as retirement or college } \\
\text { savings will be negatively impacted by coronavirus }\end{array}$ & 16 & 25 & 27 & 30 & 2 \\
\hline $\begin{array}{l}\text { You will not be able to afford testing or treatment } \\
\text { for coronavirus if you need it }\end{array}$ & 18 & 18 & 30 & 35 & * \\
\hline $\begin{array}{l}\text { You will not be able to care for a family member or } \\
\text { friend }\end{array}$ & 18 & 25 & 23 & 33 & * \\
\hline $\begin{array}{l}\text { Your existing health conditions will put you at } \\
\text { greater risk of contracting the coronavirus }\end{array}$ & 23 & 23 & 27 & 26 & 1 \\
\hline $\begin{array}{l}\text { You will be the victim of a scam about the } \\
\text { coronavirus }\end{array}$ & 12 & 16 & 26 & 46 & * \\
\hline You will feel more isolated or lonely & 12 & 24 & 31 & 33 & 1 \\
\hline You will have to postpone your retirement plans & 14 & 19 & 19 & 47 & 1 \\
\hline You will have to help neighbors financially & 6 & 16 & 26 & 47 & 4 \\
\hline You will have to help family financially & 14 & 24 & 28 & 31 & 3 \\
\hline $\begin{array}{l}\text { You will not be able to get the groceries or } \\
\text { medications you need }\end{array}$ & 16 & 25 & 24 & 31 & 3 \\
\hline $\begin{array}{l}\text { You will not be able to vote in the Presidential } \\
\text { election in November }\end{array}$ & 14 & 15 & 27 & 44 & * \\
\hline $\begin{array}{l}\text { You are concerned your Social Security benefits } \\
\text { could be reduced }\end{array}$ & 26 & 23 & 21 & 27 & 3 \\
\hline You will lose savings & 17 & 25 & 25 & 32 & 1 \\
\hline You will lose investments & 15 & 26 & 19 & 38 & 2 \\
\hline You will have to delay bill payments & 18 & 23 & 23 & 36 & $*$ \\
\hline $\begin{array}{l}\text { You might have to use retirement savings to pay } \\
\text { for necessary expenses }\end{array}$ & 16 & 28 & 24 & 31 & 2 \\
\hline $\begin{array}{l}\text { You will have to lend money to help family or } \\
\text { friends because they were impacted financially by } \\
\text { coronavirus }\end{array}$ & 15 & 19 & 33 & 31 & 3 \\
\hline $\begin{array}{l}\text { You will lose income from "gig" and side work, like } \\
\text { Uber, Airbnb, housesitting or dog-walking }\end{array}$ & 9 & 14 & 15 & 61 & 1 \\
\hline $\begin{array}{l}\text { You will lose your job, be furloughed, or have your } \\
\text { work suspended }\end{array}$ & 13 & 13 & 20 & 53 & 3 \\
\hline
\end{tabular}




\begin{tabular}{|c|c|c|c|c|c|}
\hline $\begin{array}{l}\text { Other NH } \\
05 / 29-06 / 09 / 2020\end{array}$ & $\begin{array}{c}\text { Very } \\
\text { worried }\end{array}$ & $\begin{array}{l}\text { Somewhat } \\
\text { worried }\end{array}$ & $\begin{array}{l}\text { Not very } \\
\text { worried }\end{array}$ & $\begin{array}{l}\text { Not at all } \\
\text { worried }\end{array}$ & $\begin{array}{c}\text { DON'T } \\
\text { KNOW/ } \\
\text { SKIPPED } \\
\text { ON } \\
\text { WEB/ } \\
\text { REFUSED }\end{array}$ \\
\hline $\begin{array}{l}\text { You or someone in your family will get sick from } \\
\text { the coronavirus }\end{array}$ & 27 & 25 & 20 & 28 & - \\
\hline $\begin{array}{l}\text { You or someone in your family will die from the } \\
\text { coronavirus }\end{array}$ & 21 & 17 & 37 & 26 & - \\
\hline $\begin{array}{l}\text { Your investments such as retirement or college } \\
\text { savings will be negatively impacted by coronavirus }\end{array}$ & 18 & 28 & 24 & 30 & - \\
\hline $\begin{array}{l}\text { You will not be able to afford testing or treatment } \\
\text { for coronavirus if you need it }\end{array}$ & 16 & 11 & 20 & 51 & 2 \\
\hline $\begin{array}{l}\text { You will not be able to care for a family member or } \\
\text { friend }\end{array}$ & 9 & 34 & 21 & 36 & - \\
\hline $\begin{array}{l}\text { Your existing health conditions will put you at } \\
\text { greater risk of contracting the coronavirus }\end{array}$ & 15 & 24 & 23 & 39 & - \\
\hline $\begin{array}{l}\text { You will be the victim of a scam about the } \\
\text { coronavirus }\end{array}$ & 11 & 14 & 25 & 50 & - \\
\hline You will feel more isolated or lonely & 4 & 25 & 21 & 50 & - \\
\hline You will have to postpone your retirement plans & 14 & 9 & 26 & 51 & - \\
\hline You will have to help neighbors financially & 9 & 25 & 19 & 47 & - \\
\hline You will have to help family financially & 10 & 19 & 33 & 38 & - \\
\hline $\begin{array}{l}\text { You will not be able to get the groceries or } \\
\text { medications you need }\end{array}$ & 7 & 10 & 52 & 31 & - \\
\hline $\begin{array}{l}\text { You will not be able to vote in the Presidential } \\
\text { election in November }\end{array}$ & 6 & 11 & 14 & 63 & 5 \\
\hline $\begin{array}{l}\text { You are concerned your Social Security benefits } \\
\text { could be reduced }\end{array}$ & 13 & 28 & 18 & 41 & - \\
\hline You will lose savings & 10 & 23 & 36 & 31 & - \\
\hline You will lose investments & 22 & 25 & 23 & 30 & - \\
\hline You will have to delay bill payments & 10 & 4 & 36 & 49 & 2 \\
\hline $\begin{array}{l}\text { You might have to use retirement savings to pay } \\
\text { for necessary expenses }\end{array}$ & 11 & 14 & 24 & 52 & - \\
\hline $\begin{array}{l}\text { You will have to lend money to help family or } \\
\text { friends because they were impacted financially by } \\
\text { coronavirus }\end{array}$ & 8 & 17 & 41 & 34 & - \\
\hline $\begin{array}{l}\text { You will lose income from "gig" and side work, like } \\
\text { Uber, Airbnb, housesitting or dog-walking }\end{array}$ & 6 & 10 & 16 & 69 & - \\
\hline $\begin{array}{l}\text { You will lose your job, be furloughed, or have your } \\
\text { work suspended }\end{array}$ & 11 & 6 & 19 & 64 & - \\
\hline
\end{tabular}


AARP 50+ Coronavirus Tracker: Wave 5 Oversample

If employed

Q7. In what ways, if any, has the coronavirus affected your job or income?

[ITEMS RANDOMIZED]

\begin{tabular}{|l|c|c|c|c|c|}
\hline $\begin{array}{l}\text { NORC } \\
\text { 05/29-06/09/2020 }\end{array}$ & Total & White NH & $\begin{array}{c}\text { African } \\
\text { American } \\
\mathrm{NH}\end{array}$ & Hispanic & Other NH \\
\hline $\begin{array}{l}\text { You lost income due to a workplace closure } \\
\text { or reduced hours }\end{array}$ & 29 & 29 & 28 & 29 & $*$ \\
\hline $\begin{array}{l}\text { You had to put yourself at risk of exposure to } \\
\text { coronavirus because you can't afford to stay } \\
\text { home and miss work }\end{array}$ & 15 & 12 & 20 & 16 & $*$ \\
\hline $\begin{array}{l}\text { You changed your work routine such as } \\
\text { working from home }\end{array}$ & 26 & 32 & 25 & 17 & $*$ \\
\hline You lost a job or had your work suspended & 16 & 15 & 12 & 22 & $*$ \\
\hline $\begin{array}{l}\text { You took on a loan or additional debt to pay } \\
\text { for necessary expenses }\end{array}$ & 5 & 5 & 7 & 5 & $*$ \\
\hline Your income increased & 6 & 4 & 3 & 9 & $*$ \\
\hline Your income was not affected at all & 45 & 49 & 49 & 35 & $*$ \\
\hline DON'T KNOW/ SKIPPED ON WEB/ REFUSED & 1 & 1 & 1 & $*$ & $*$ \\
\hline
\end{tabular}

Note: Base sizes too small to report percentages for Other $\mathrm{NH}$.

Q8. How satisfied are you, if at all, with the quality of your social relationships during the coronavirus pandemic?

\begin{tabular}{|l|c|c|c|c|c|}
\hline $\begin{array}{l}\text { NORC } \\
\text { 05/29-06/09/2020 }\end{array}$ & Total & White NH & $\begin{array}{c}\text { African } \\
\text { American } \\
\mathrm{NH}\end{array}$ & Hispanic & Other NH \\
\hline Very satisfied & 23 & 22 & 27 & 22 & 27 \\
\hline Somewhat satisfied & 49 & 54 & 46 & 42 & 45 \\
\hline Not very satisfied & 22 & 20 & 16 & 30 & 24 \\
\hline Not at all satisfied & 6 & 4 & 10 & 5 & 4 \\
\hline DON'T KNOW/ SKIPPED ON WEB/ REFUSED & 1 & $*$ & 1 & 1 & - \\
\hline
\end{tabular}


Q9. How often do you...

[ITEMS RANDOMIZED]

\begin{tabular}{|l|c|c|c|c|}
\hline $\begin{array}{l}\text { Total } \\
\text { 05/29-06/09/2020 }\end{array}$ & & & & $\begin{array}{c}\text { DON'T } \\
\text { KNOW/ } \\
\text { SKIPPED } \\
\text { ON WEB/ } \\
\text { REFUSED }\end{array}$ \\
\hline Feel you lack companionship & Hardly ever & $\begin{array}{c}\text { Some of } \\
\text { the time }\end{array}$ & Often & \\
\hline Feel left out & 53 & 36 & 11 & 1 \\
\hline Feel isolated from others & 59 & 31 & 9 & 1 \\
\hline
\end{tabular}

$N=1,798$

\begin{tabular}{|l|c|c|c|c|}
\hline White $\mathrm{NH}$ & & & & $\begin{array}{c}\text { DON'T } \\
\text { KNOW/ } \\
\text { 05/29-06/09/2020 }\end{array}$ \\
& HKIPPED \\
& Hardly ever & $\begin{array}{c}\text { Some of } \\
\text { the time }\end{array}$ & & $\begin{array}{c}\text { ON WEB/ } \\
\text { REFUSED }\end{array}$ \\
\hline Feel you lack companionship & 55 & 34 & 11 & 1 \\
\hline Feel left out & 58 & 33 & 8 & 1 \\
\hline Feel isolated from others & 43 & 42 & 14 & 1 \\
\hline
\end{tabular}

$N=906$

\begin{tabular}{|l|c|c|c|c|}
\hline $\begin{array}{l}\text { African American NH } \\
\text { 05/29-06/09/2020 }\end{array}$ & & & & $\begin{array}{c}\text { DON'T } \\
\text { KNOW/ } \\
\text { SKIPPED } \\
\text { ON WEB/ } \\
\text { REFUSED }\end{array}$ \\
\hline Feel you lack companionship & Hardly ever & $\begin{array}{c}\text { Some of } \\
\text { the time }\end{array}$ & Often & 12 \\
\hline Feel left out & 54 & 33 & 10 & 3 \\
\hline Feel isolated from others & 57 & 31 & 9 & 1 \\
\hline
\end{tabular}

$N=401$

\begin{tabular}{|l|c|c|c|c|}
\hline $\begin{array}{l}\text { Hispanic } \\
\text { 05/29-06/09/2020 }\end{array}$ & & & & $\begin{array}{c}\text { DON'T } \\
\text { KNOW/ } \\
\text { SKIPPED } \\
\text { ON WEB/ } \\
\text { REFUSED }\end{array}$ \\
\hline Feel you lack companionship & Hardly ever & $\begin{array}{c}\text { Some of } \\
\text { the time }\end{array}$ & $\begin{array}{c} \\
\text { Often }\end{array}$ & $\begin{array}{c}\text { REF } \\
\text { Feel left out }\end{array}$ \\
\hline Feel isolated from others & 45 & 45 & 10 & 1 \\
\hline$N=444$ & 41 & 42 & 10 & 1 \\
\hline
\end{tabular}

$N=444$ 


\begin{tabular}{|l|c|c|c|c|}
\hline $\begin{array}{l}\text { Other NH } \\
\text { 05/29-06/09/2020 }\end{array}$ & & & & $\begin{array}{c}\text { DON'T } \\
\text { KNOW/ } \\
\text { SKIPPED } \\
\text { ON WEB/ } \\
\text { REFUSED }\end{array}$ \\
\hline Feel you lack companionship & Hardly ever & $\begin{array}{c}\text { Some of } \\
\text { the time }\end{array}$ & Often & \\
\hline Feel left out & 72 & 18 & 11 & - \\
\hline Feel isolated from others & 61 & 30 & 9 & - \\
\hline
\end{tabular}

$N=47$

Q10. Do you feel more lonely now than before the coronavirus pandemic, less lonely now, or has there been no change?

\begin{tabular}{|l|c|c|c|c|c|}
\hline $\begin{array}{l}\text { NORC } \\
\text { 05/29-06/09/2020 }\end{array}$ & Total & White NH & $\begin{array}{c}\text { African } \\
\text { American } \\
\mathrm{NH}\end{array}$ & Hispanic & Other $\mathrm{NH}$ \\
\hline More lonely now & 31 & 36 & 27 & 25 & 25 \\
\hline Less lonely now & 5 & 3 & 9 & 8 & - \\
\hline No change & 64 & 61 & 63 & 67 & 74 \\
\hline DON'T KNOW/ SKIPPED ON WEB/ REFUSED & $*$ & - & 1 & $*$ & 2 \\
\hline
\end{tabular}

Q11. In general, are you feeling more connected to any of the following people in your life during the coronavirus pandemic, less connected, having no change in connection, or do you not have this type of person in your life?

[ITEMS RANDOMIZED]

\begin{tabular}{|l|c|c|c|c|c|}
\hline $\begin{array}{l}\text { Total } \\
\text { 05/29-06/09/2020 }\end{array}$ & $\begin{array}{c}\text { More } \\
\text { connected }\end{array}$ & $\begin{array}{c}\text { Less } \\
\text { connected }\end{array}$ & $\begin{array}{c}\text { No change in } \\
\text { connectedness }\end{array}$ & $\begin{array}{c}\text { Don't have } \\
\text { this type of } \\
\text { person in my } \\
\text { life }\end{array}$ & $\begin{array}{c}\text { DON'T } \\
\text { KNOW/ } \\
\text { SKIPPED ON } \\
\text { WEB/ } \\
\text { REFUSED }\end{array}$ \\
\hline Spouse & 22 & 5 & 34 & 38 & 1 \\
\hline Children who are under 18 years old & 9 & 12 & 21 & 57 & 1 \\
\hline Children who are over 18 years old & 16 & 21 & 42 & 20 & 1 \\
\hline Grandchildren & 12 & 24 & 27 & 37 & 1 \\
\hline Parent(s) & 10 & 11 & 22 & 56 & 1 \\
\hline Grandparent(s) & 2 & 3 & 7 & 86 & 2 \\
\hline Sibling(s) & 17 & 19 & 50 & 13 & 1 \\
\hline Friend(s) & 13 & 41 & 42 & 4 & $*$ \\
\hline Neighbor(s) & 13 & 24 & 58 & 5 & 1 \\
\hline Co-Worker(s) & 5 & 21 & 26 & 46 & 1 \\
\hline
\end{tabular}

$N=1,798$ 


\begin{tabular}{|l|c|c|c|c|c|}
\hline $\begin{array}{l}\text { White } \mathrm{NH} \\
\text { 05/29-06/09/2020 }\end{array}$ & $\begin{array}{c}\text { More } \\
\text { connected }\end{array}$ & $\begin{array}{c}\text { Less } \\
\text { connected }\end{array}$ & $\begin{array}{c}\text { No change in } \\
\text { connectedness }\end{array}$ & $\begin{array}{c}\text { Don't have } \\
\text { this type of } \\
\text { person in my } \\
\text { life }\end{array}$ & $\begin{array}{c}\text { DON'T } \\
\text { KNOW/ } \\
\text { SKIPPED ON } \\
\text { WEB/ } \\
\text { REFUSED }\end{array}$ \\
\hline Spouse & 24 & 3 & 40 & 33 & $*$ \\
\hline Children who are under 18 years old & 6 & 12 & 18 & 64 & 1 \\
\hline Children who are over 18 years old & 14 & 25 & 40 & 21 & $*$ \\
\hline Grandchildren & 8 & 26 & 24 & 42 & 1 \\
\hline Parent(s) & 6 & 12 & 20 & 61 & 1 \\
\hline Grandparent(s) & 1 & 2 & 5 & 91 & 2 \\
\hline Sibling(s) & 14 & 20 & 50 & 14 & 1 \\
\hline Friend(s) & 9 & 49 & 39 & 2 & - \\
\hline Neighbor(s) & 12 & 23 & 62 & 4 & $*$ \\
\hline Co-Worker(s) & 4 & 22 & 23 & 50 & 1 \\
\hline
\end{tabular}

$$
N=906
$$

\begin{tabular}{|l|c|c|c|c|c|}
\hline $\begin{array}{l}\text { African American NH } \\
\text { 05/29-06/09/2020 }\end{array}$ & $\begin{array}{c}\text { More } \\
\text { connected }\end{array}$ & $\begin{array}{c}\text { Less } \\
\text { connected }\end{array}$ & $\begin{array}{c}\text { No change in } \\
\text { connectedness }\end{array}$ & $\begin{array}{c}\text { Don't have } \\
\text { this type of } \\
\text { person in my } \\
\text { life }\end{array}$ & $\begin{array}{c}\text { DON'T } \\
\text { KNOW/ } \\
\text { SKIPPED ON } \\
\text { WEB/ } \\
\text { REFUSED }\end{array}$ \\
\hline Spouse & 16 & 6 & 27 & 48 & 3 \\
\hline Children who are under 18 years old & 12 & 13 & 27 & 48 & 1 \\
\hline Children who are over 18 years old & 21 & 19 & 41 & 19 & 1 \\
\hline Grandchildren & 20 & 19 & 30 & 30 & 1 \\
\hline Parent(s) & 12 & 9 & 20 & 58 & 1 \\
\hline Grandparent(s) & 4 & 5 & 11 & 80 & 1 \\
\hline Sibling(s) & 25 & 15 & 48 & 11 & 2 \\
\hline Friend(s) & 20 & 30 & 45 & 5 & $*$ \\
\hline Neighbor(s) & 15 & 25 & 53 & 6 & 2 \\
\hline Co-Worker(s) & 10 & 22 & 26 & 41 & 1 \\
\hline
\end{tabular}

$N=401$ 


\begin{tabular}{|l|c|c|c|c|c|}
\hline $\begin{array}{l}\text { Hispanic } \\
\text { 05/29-06/09/2020 }\end{array}$ & $\begin{array}{c}\text { More } \\
\text { connected }\end{array}$ & $\begin{array}{c}\text { Less } \\
\text { connected }\end{array}$ & $\begin{array}{c}\text { No change in } \\
\text { connectedness }\end{array}$ & $\begin{array}{c}\text { Don't have } \\
\text { this type of } \\
\text { person in my } \\
\text { life }\end{array}$ & $\begin{array}{c}\text { DON'T } \\
\text { KNOW/ } \\
\text { SKIPPED ON } \\
\text { WEB/ } \\
\text { REFUSED }\end{array}$ \\
\hline Spouse & 22 & 6 & 32 & 40 & $*$ \\
\hline Children who are under 18 years old & 14 & 12 & 20 & 53 & 2 \\
\hline Children who are over 18 years old & 17 & 15 & 48 & 20 & 1 \\
\hline Grandchildren & 12 & 24 & 27 & 35 & 2 \\
\hline Parent(s) & 15 & 12 & 27 & 45 & 2 \\
\hline Grandparent(s) & 2 & 6 & 9 & 81 & 2 \\
\hline Sibling(s) & 17 & 20 & 49 & 13 & $*$ \\
\hline Friend(s) & 14 & 36 & 44 & 6 & $*$ \\
\hline Neighbor(s) & 13 & 25 & 56 & 5 & 1 \\
\hline Co-Worker(s) & 4 & 19 & 31 & 46 & $*$ \\
\hline
\end{tabular}

\begin{tabular}{|l|c|c|c|c|c|}
\hline $\begin{array}{l}\text { Other NH } \\
\text { 05/29-06/09/2020 }\end{array}$ & $\begin{array}{c}\text { More } \\
\text { connected }\end{array}$ & $\begin{array}{c}\text { Less } \\
\text { connected }\end{array}$ & $\begin{array}{c}\text { No change in } \\
\text { connectedness }\end{array}$ & $\begin{array}{c}\text { Don't have } \\
\text { this type of } \\
\text { person in my } \\
\text { life }\end{array}$ & $\begin{array}{c}\text { DON'T } \\
\text { KNOW/ } \\
\text { SKIPPED ON } \\
\text { WEB/ } \\
\text { REFUSED }\end{array}$ \\
\hline Spouse & 21 & 5 & 30 & 42 & 2 \\
\hline Children who are under 18 years old & 13 & 8 & 23 & 56 & - \\
\hline Children who are over 18 years old & 10 & 18 & 46 & 24 & 2 \\
\hline Grandchildren & 11 & 17 & 42 & 31 & - \\
\hline Parent(s) & 15 & 9 & 26 & 50 & - \\
\hline Grandparent(s) & - & 1 & 7 & 89 & 2 \\
\hline Sibling(s) & 13 & 10 & 63 & 13 & - \\
\hline Friend(s) & 9 & 36 & 53 & - & 2 \\
\hline Neighbor(s) & 14 & 30 & 54 & 1 & 2 \\
\hline Co-Worker(s) & 2 & 25 & 36 & 38 & - \\
\hline
\end{tabular}

If have grandchild(ren)

Q12. During the coronavirus pandemic, have you been able to see your grandchild(ren) more, less, or about the same amount as prior to the pandemic?

\begin{tabular}{|l|c|c|c|c|c|}
\hline $\begin{array}{l}\text { NORC } \\
\text { 05/29-06/09/2020 }\end{array}$ & Total & White NH & $\begin{array}{c}\text { African } \\
\text { American } \\
\text { NH }\end{array}$ & Hispanic & Other NH \\
\hline More & 8 & 8 & 10 & 8 & - \\
\hline Less & 54 & 60 & 42 & 56 & 46 \\
\hline Same & 38 & 32 & 48 & 36 & 52 \\
\hline DON'T KNOW/ SKIPPED ON WEB/ REFUSED & 1 & $*$ & $*$ & $*$ & 3 \\
\hline
\end{tabular}


Q14. Which of the following groups or organizations have helped you connect with others during the coronavirus pandemic?

Please select all that apply.

[ITEMS RANDOMIZED]

\begin{tabular}{|l|c|c|c|c|c|}
\hline $\begin{array}{l}\text { NORC } \\
\text { 05/29-06/09/2020 }\end{array}$ & Total & White NH & $\begin{array}{c}\text { African } \\
\text { American } \\
\mathrm{NH}\end{array}$ & Hispanic & Other NH \\
\hline $\begin{array}{l}\text { A faith-based organization (such as churches, } \\
\text { temples, synagogues, and mosques) }\end{array}$ & 31 & 28 & 46 & 24 & 36 \\
\hline $\begin{array}{l}\text { A community organization (such as a Parent- } \\
\text { Teacher association, sports club, } \\
\text { neighborhood association) }\end{array}$ & 9 & 6 & 15 & 8 & 9 \\
\hline An alumni association & 3 & 2 & 5 & 2 & 2 \\
\hline An educational institution & 5 & 4 & 7 & 4 & 9 \\
\hline A political organization & 4 & 4 & 2 & 5 & 3 \\
\hline $\begin{array}{l}\text { A service organization (such as Elks, Kiwanis, } \\
\text { Lions, or Rotary clubs) }\end{array}$ & 4 & 5 & 4 & 2 & 2 \\
\hline A veterans' group & 3 & 3 & 4 & 4 & 3 \\
\hline Other (specify): & 30 & 30 & 29 & 30 & 30 \\
\hline DON'T KNOW/ SKIPPED ON WEB/ REFUSED & 28 & 32 & 18 & 30 & 23 \\
\hline
\end{tabular}

Q15. In the past week, how many days have there been when you've gone without speaking to anyone (either through phone, video, or in-person)?

\begin{tabular}{|l|c|c|c|c|c|}
\hline $\begin{array}{l}\text { NORC } \\
\text { 55/29-06/09/2020 }\end{array}$ & Total & White NH & $\begin{array}{c}\text { African } \\
\text { American } \\
\mathrm{NH}\end{array}$ & Hispanic & Other NH \\
\hline None & 81 & 86 & 79 & 75 & 75 \\
\hline 1 & 4 & 3 & 4 & 7 & 3 \\
\hline 2 & 4 & 4 & 4 & 4 & 9 \\
\hline 3 & 4 & 4 & 6 & 4 & 5 \\
\hline 4 & 3 & 2 & 2 & 5 & 8 \\
\hline 5 & 2 & 1 & 2 & 2 & 1 \\
\hline 6 & $*$ & $*$ & 1 & $*$ & - \\
\hline 7 & 2 & 1 & 2 & 3 & - \\
\hline DON'T KNOW/ SKIPPED ON WEB/ REFUSED & $*$ & $*$ & 1 & 1 & - \\
\hline
\end{tabular}


Q16. Which of the following activities have you engaged in to connect with people during the coronavirus pandemic?

Please select all that apply.

[ITEMS RANDOMIZED]

\begin{tabular}{|l|c|c|c|c|c|}
\hline $\begin{array}{l}\text { NORC } \\
\text { 05/29-06/09/2020 }\end{array}$ & Total & White NH & $\begin{array}{c}\text { African } \\
\text { American } \\
\mathrm{NH}\end{array}$ & Hispanic & Other NH \\
\hline Video chats & 49 & 51 & 53 & 45 & 45 \\
\hline Telephone calls & 89 & 90 & 92 & 86 & 90 \\
\hline Text messages & 82 & 86 & 78 & 78 & 74 \\
\hline Email exchanges & 59 & 71 & 51 & 44 & 54 \\
\hline Letter exchanges & 11 & 11 & 14 & 10 & 6 \\
\hline Live virtual events & 25 & 27 & 27 & 20 & 20 \\
\hline Virtual or remote volunteering & 6 & 6 & 7 & 4 & 3 \\
\hline None of the above & 2 & 2 & 3 & 1 & 1 \\
\hline DON'T KNOW/ SKIPPED ON WEB/ REFUSED & $*$ & - & 1 & 1 & - \\
\hline
\end{tabular}

If engaged in any activities to connect with people Q17. Was the first time you engaged in this activity during the pandemic?

\begin{tabular}{|l|c|c|c|c|}
\hline Total & & & $\begin{array}{c}\text { DON'T } \\
\text { KNOW/ } \\
\text { SKIPPED } \\
\text { O5/29-06/09/2020 }\end{array}$ & Yes WEB/ \\
REFUSED & \\
\hline Video chats & 32 & 67 & 1 & $N=962$ \\
\hline Telephone calls & 12 & 87 & 1 & $N=1,601$ \\
\hline Text messages & 11 & 87 & 2 & $N=1,535$ \\
\hline Email exchanges & 9 & 90 & 2 & $N=1,167$ \\
\hline Letter exchanges & 9 & 86 & 5 & $N=197$ \\
\hline Live virtual events & 50 & 48 & 2 & $N=504$ \\
\hline Virtual or remote volunteering & 53 & 46 & $*$ & $N=112$ \\
\hline
\end{tabular}




\begin{tabular}{|c|c|c|c|c|}
\hline $\begin{array}{l}\text { White NH } \\
05 / 29-06 / 09 / 2020\end{array}$ & Yes & No & $\begin{array}{c}\text { DON'T } \\
\text { KNOW/ } \\
\text { SKIPPED } \\
\text { ON WEB/ } \\
\text { REFUSED }\end{array}$ & \\
\hline Video chats & 34 & 65 & 1 & $N=472$ \\
\hline Telephone calls & 6 & 93 & 1 & $N=813$ \\
\hline Text messages & 5 & 93 & 3 & $N=797$ \\
\hline Email exchanges & 4 & 94 & 2 & $N=660$ \\
\hline Letter exchanges & 10 & 89 & 1 & $N=91$ \\
\hline Live virtual events & 54 & 45 & 2 & $N=257$ \\
\hline Virtual or remote volunteering & 50 & 51 & - & $N=52$ \\
\hline
\end{tabular}

\begin{tabular}{|l|c|c|c|c|}
\hline $\begin{array}{l}\text { African American NH } \\
\text { 05/29-06/09/2020 }\end{array}$ & & & $\begin{array}{c}\text { DON'T } \\
\text { KNOW/ } \\
\text { SKIPPED } \\
\text { ON WEB/ } \\
\text { REFUSED }\end{array}$ & \\
\hline Video chats & Yes & No & \\
\hline Telephone calls & 25 & 74 & 1 & $N=222$ \\
\hline Text messages & 16 & 84 & 1 & $N=367$ \\
\hline Email exchanges & 16 & 83 & 2 & $N=330$ \\
\hline Letter exchanges & 19 & 80 & 1 & $N=226$ \\
\hline Live virtual events & 11 & 77 & 12 & $N=55$ \\
\hline Virtual or remote volunteering & 45 & 52 & 3 & $N=121$ \\
\hline
\end{tabular}

\begin{tabular}{|l|c|c|c|c|}
\hline $\begin{array}{l}\text { Hispanic } \\
\text { 05/29-06/09/2020 }\end{array}$ & & & $\begin{array}{c}\text { DON'T } \\
\text { KNOW/ } \\
\text { SKIPPED } \\
\text { ON WEB/ } \\
\text { REFUSED }\end{array}$ & \\
\hline Video chats & Yes & No & \\
\hline Telephone calls & 36 & 64 & $*$ & $N=245$ \\
\hline Text messages & 21 & 79 & 1 & $N=381$ \\
\hline Email exchanges & 19 & 79 & 1 & $N=370$ \\
\hline Letter exchanges & 13 & 86 & 1 & $N=249$ \\
\hline Live virtual events & 8 & 89 & 3 & $N=47$ \\
\hline Virtual or remote volunteering & 44 & 54 & 2 & $N=113$ \\
\hline
\end{tabular}

Note: Base sizes too small to report percentages on Virtual or remote volunteering. 


\begin{tabular}{|l|c|c|c|c|}
\hline $\begin{array}{l}\text { Other NH } \\
\text { 05/29-06/09/2020 }\end{array}$ & & & $\begin{array}{c}\text { DON'T } \\
\text { KNOW/ } \\
\text { SKIPPED } \\
\text { ON WEB/ } \\
\text { REFUSED }\end{array}$ & \\
\hline Video chats & Yes & No & $*$ \\
\hline Telephone calls & $*$ & $*$ & $*$ & $N=23$ \\
\hline Text messages & 11 & 84 & 6 & $N=40$ \\
\hline Email exchanges & 9 & 86 & 5 & $N=38$ \\
\hline Letter exchanges & 13 & 85 & 2 & $N=32$ \\
\hline Live virtual events & $*$ & $*$ & $*$ & $N=4$ \\
\hline Virtual or remote volunteering & $*$ & $*$ & $*$ & $N=13$ \\
\hline
\end{tabular}

Note: Base sizes too small to report percentages Video chats, Letter exchanges, and Virtual or remote volunteering.

If engaged in any activities to connect with people

Q18. Compared to before the coronavirus pandemic, do you now do more, less, or the same amount of the following activities?

\begin{tabular}{|l|c|c|c|c|c|}
\hline $\begin{array}{l}\text { Total } \\
\text { 05/29-06/09/2020 }\end{array}$ & $\begin{array}{c}\text { I do more } \\
\text { now }\end{array}$ & $\begin{array}{c}\text { I do less } \\
\text { now }\end{array}$ & $\begin{array}{c}\text { I do the } \\
\text { same } \\
\text { amount } \\
\text { now }\end{array}$ & $\begin{array}{c}\text { DON'T } \\
\text { KNOW/ } \\
\text { SKIPPED } \\
\text { ON WEB/ } \\
\text { REFUSED }\end{array}$ & \\
\hline Video chats & 68 & 4 & 28 & 1 & $N=961$ \\
\hline Telephone calls & 35 & 5 & 59 & 1 & $N=1,598$ \\
\hline Text messages & 39 & 4 & 56 & $*$ & $N=1,532$ \\
\hline Email exchanges & 29 & 5 & 66 & $*$ & $N=1,168$ \\
\hline Letter exchanges & 25 & 4 & 66 & 5 & $N=198$ \\
\hline Live virtual events & 75 & 7 & 17 & 1 & $N=504$ \\
\hline Virtual or remote volunteering & 56 & 9 & 35 & $*$ & $N=112$ \\
\hline
\end{tabular}

\begin{tabular}{|l|c|c|c|c|c|}
\hline $\begin{array}{l}\text { White NH } \\
\text { 05/29-06/09/2020 }\end{array}$ & $\begin{array}{c}\text { I do more } \\
\text { now }\end{array}$ & $\begin{array}{c}\text { I do less } \\
\text { now }\end{array}$ & $\begin{array}{c}\text { I do the } \\
\text { same } \\
\text { amount now }\end{array}$ & $\begin{array}{c}\text { KNOW/ } \\
\text { SKIPPED } \\
\text { ON WEB/ } \\
\text { REFUSED }\end{array}$ & \\
\hline Video chats & 74 & 3 & 22 & $*$ & $N=472$ \\
\hline Telephone calls & 31 & 3 & 66 & $*$ & $N=813$ \\
\hline Text messages & 34 & 4 & 62 & $*$ & $N=797$ \\
\hline Email exchanges & 25 & 5 & 69 & $*$ & $N=660$ \\
\hline Letter exchanges & 33 & 2 & 65 & - & $N=91$ \\
\hline Live virtual events & 82 & 3 & 15 & $*$ & $N=257$ \\
\hline Virtual or remote volunteering & 61 & 9 & 30 & - & $N=52$ \\
\hline
\end{tabular}




\begin{tabular}{|l|c|c|c|c|c|}
\hline $\begin{array}{l}\text { African American NH } \\
\text { 05/29-06/09/2020 }\end{array}$ & $\begin{array}{c}\text { I do more } \\
\text { now }\end{array}$ & $\begin{array}{c}\text { I do less } \\
\text { now }\end{array}$ & $\begin{array}{c}\text { I do the } \\
\text { same } \\
\text { amount now }\end{array}$ & $\begin{array}{c}\text { KNOW/ } \\
\text { SKIPPED } \\
\text { ON WEB/ } \\
\text { REFUSED }\end{array}$ & \\
\hline Video chats & 55 & 6 & 37 & 2 & $N=221$ \\
\hline Telephone calls & 43 & 5 & 51 & $*$ & $N=365$ \\
\hline Text messages & 44 & 6 & 49 & $*$ & $N=329$ \\
\hline Email exchanges & 37 & 6 & 57 & $*$ & $N=227$ \\
\hline Letter exchanges & 11 & 10 & 63 & 17 & $N=56$ \\
\hline Live virtual events & 70 & 9 & 19 & 3 & $N=121$ \\
\hline Virtual or remote volunteering & 47 & 12 & 41 & - & $N=31$ \\
\hline
\end{tabular}

\begin{tabular}{|l|c|c|c|c|c|}
\hline $\begin{array}{l}\text { Hispanic } \\
\text { 05/29-06/09/2020 }\end{array}$ & $\begin{array}{c}\text { I do more } \\
\text { now }\end{array}$ & $\begin{array}{c}\text { I do less } \\
\text { now }\end{array}$ & $\begin{array}{c}\text { DON'T } \\
\text { I do the } \\
\text { same } \\
\text { amount now }\end{array}$ & $\begin{array}{c}\text { KNOW/ } \\
\text { SKIPPED } \\
\text { ON WEB/ } \\
\text { REFUSED }\end{array}$ & \\
\hline Video chats & 68 & 4 & 28 & - & $N=245$ \\
\hline Telephone calls & 37 & 8 & 54 & 1 & $N=380$ \\
\hline Text messages & 43 & 4 & 53 & 1 & $N=368$ \\
\hline Email exchanges & 35 & 5 & 59 & $*$ & $N=249$ \\
\hline Letter exchanges & 27 & 2 & 71 & - & $N=47$ \\
\hline Live virtual events & 59 & 19 & 20 & 2 & $N=113$ \\
\hline Virtual or remote volunteering & $*$ & $*$ & $*$ & $*$ & $N=27$ \\
\hline
\end{tabular}

Note: Base sizes too small to report percentages on Virtual or remote volunteering.

\begin{tabular}{|l|c|c|c|c|c|}
\hline $\begin{array}{l}\text { Other NH } \\
\text { 05/29-06/09/2020 }\end{array}$ & $\begin{array}{c}\text { I do more } \\
\text { now }\end{array}$ & $\begin{array}{c}\text { I do less } \\
\text { now }\end{array}$ & $\begin{array}{c}\text { DON'T } \\
\text { I do the } \\
\text { same } \\
\text { amount now }\end{array}$ & $\begin{array}{c}\text { KNOW/ } \\
\text { SKIPPED } \\
\text { ON WEB/ } \\
\text { REFUSED }\end{array}$ & \\
\hline Video chats & $*$ & $*$ & $*$ & $*$ & $N=23$ \\
\hline Telephone calls & 32 & 3 & 58 & 6 & $N=40$ \\
\hline Text messages & 52 & - & 48 & - & $N=38$ \\
\hline Email exchanges & 18 & 2 & 80 & - & $N=32$ \\
\hline Letter exchanges & $*$ & $*$ & $*$ & $*$ & $N=4$ \\
\hline Live virtual events & $*$ & $*$ & $*$ & $*$ & $N=13$ \\
\hline Virtual or remote volunteering & $*$ & $*$ & $*$ & $*$ & $N=2$ \\
\hline
\end{tabular}

Note: Base sizes too small to report percentages for Video chats, Letter exchanges, Live Virtual events and Virtual or remote volunteering. 
AARP 50+ Coronavirus Tracker: Wave 5 Oversample

If engaged in any activities to connect with people

Q19. When coronavirus restrictions are lifted, do you anticipate doing more, less, or the same amount of the following activities as you are doing now during the pandemic?

\begin{tabular}{|l|c|c|c|c|c|}
\hline $\begin{array}{l}\text { Total } \\
\text { 05/29-06/09/2020 }\end{array}$ & $\begin{array}{c}\text { I anticipate doing } \\
\text { more when } \\
\text { restrictions are } \\
\text { lifted }\end{array}$ & $\begin{array}{c}\text { I anticipate doing } \\
\text { less when } \\
\text { restrictions are } \\
\text { lifted }\end{array}$ & $\begin{array}{c}\text { I anticipate doing } \\
\text { the same amount } \\
\text { when restrictions } \\
\text { are lifted }\end{array}$ & $\begin{array}{c}\text { DON'T } \\
\text { KNOW/ } \\
\text { SKIPPED ON } \\
\text { WEB/ } \\
\text { REFUSED }\end{array}$ & \\
\hline Video chats & 10 & 38 & 51 & 1 & $N=961$ \\
\hline Telephone calls & 10 & 15 & 74 & 1 & $N=1,598$ \\
\hline Text messages & 8 & 12 & 80 & $*$ & $N=1,532$ \\
\hline Email exchanges & 7 & 14 & 79 & $*$ & $N=1,168$ \\
\hline Letter exchanges & 7 & 25 & 38 & 1 & $N=198$ \\
\hline Live virtual events & 9 & 53 & 43 & - & $N=504$ \\
\hline $\begin{array}{l}\text { Virtual or remote } \\
\text { volunteering }\end{array}$ & 3 & 54 & & & $N=112$ \\
\hline
\end{tabular}

\begin{tabular}{|l|c|c|c|c|c|}
\hline $\begin{array}{l}\text { White } \mathrm{NH} \\
\text { 05/29-06/09/2020 }\end{array}$ & $\begin{array}{c}\text { I anticipate doing } \\
\text { more when } \\
\text { restrictions are } \\
\text { lifted }\end{array}$ & $\begin{array}{c}\text { I anticipate doing } \\
\text { less when } \\
\text { restrictions are } \\
\text { lifted }\end{array}$ & $\begin{array}{c}\text { I anticipate doing } \\
\text { the same amount } \\
\text { when restrictions } \\
\text { are lifted }\end{array}$ & $\begin{array}{c}\text { DON'T } \\
\text { KNOW/ } \\
\text { SKIPPED ON } \\
\text { WEB/ } \\
\text { REFUSED }\end{array}$ & ( \\
\hline Video chats & 8 & 48 & 44 & 1 & $N=472$ \\
\hline Telephone calls & 7 & 14 & 79 & $*$ & $N=813$ \\
\hline Text messages & 5 & 11 & 84 & 1 & $N=797$ \\
\hline Email exchanges & 4 & 12 & 83 & 1 & $N=660$ \\
\hline Letter exchanges & 3 & 27 & 34 & 1 & $N=257$ \\
\hline Live virtual events & 7 & 59 & 34 & - & $N=52$ \\
\hline $\begin{array}{l}\text { Virtual or remote } \\
\text { volunteering }\end{array}$ & 3 & 63 & & & $*$ \\
\hline
\end{tabular}




\begin{tabular}{|l|c|c|c|c|c|}
\hline $\begin{array}{l}\text { African American NH } \\
\text { 05/29-06/09/2020 }\end{array}$ & $\begin{array}{c}\text { I anticipate doing } \\
\text { more when } \\
\text { restrictions are } \\
\text { lifted }\end{array}$ & $\begin{array}{c}\text { I anticipate doing } \\
\text { less when } \\
\text { restrictions are } \\
\text { lifted }\end{array}$ & $\begin{array}{c}\text { I anticipate doing } \\
\text { the same amount } \\
\text { when restrictions } \\
\text { are lifted }\end{array}$ & $\begin{array}{c}\text { DON'T } \\
\text { KNOW/ } \\
\text { SKIPPED ON } \\
\text { WEB/ } \\
\text { REFUSED }\end{array}$ & * \\
\hline Video chats & 12 & 23 & 65 & 71 & $N=221$ \\
\hline Telephone calls & 17 & 12 & 74 & 1 & $N=365$ \\
\hline Text messages & 13 & 13 & 70 & 1 & $N=329$ \\
\hline Email exchanges & 11 & 18 & 64 & - & $N=227$ \\
\hline Letter exchanges & 9 & 47 & 49 & $*$ & $N=121$ \\
\hline Live virtual events & 11 & 49 & 49 & - & $N=31$ \\
\hline $\begin{array}{l}\text { Virtual or remote } \\
\text { volunteering }\end{array}$ & 1 & & & & $*$ \\
\hline
\end{tabular}

\begin{tabular}{|l|c|c|c|c|c|}
\hline $\begin{array}{l}\text { Hispanic } \\
\text { 05/29-06/09/2020 }\end{array}$ & $\begin{array}{c}\text { I anticipate doing } \\
\text { more when } \\
\text { restrictions are } \\
\text { lifted }\end{array}$ & $\begin{array}{c}\text { I anticipate doing } \\
\text { less when } \\
\text { restrictions are } \\
\text { lifted }\end{array}$ & $\begin{array}{c}\text { I anticipate doing } \\
\text { the same amount } \\
\text { when restrictions } \\
\text { are lifted }\end{array}$ & $\begin{array}{c}\text { DON'T } \\
\text { KNOW/ } \\
\text { SKIPED ON } \\
\text { WEB/ } \\
\text { REFUSED }\end{array}$ & -2 \\
\hline Video chats & 14 & 36 & 50 & 3 & $N=245$ \\
\hline Telephone calls & 9 & 21 & 67 & 1 & $N=380$ \\
\hline Text messages & 9 & 13 & 77 & $*$ & $N=249$ \\
\hline Email exchanges & 12 & 16 & 68 & - & $N=47$ \\
\hline Letter exchanges & 14 & 18 & 31 & 1 & $N=113$ \\
\hline Live virtual events & 14 & 54 & $*$ & $*$ & $N=27$ \\
\hline $\begin{array}{l}\text { Virtual or remote } \\
\text { volunteering }\end{array}$ & $*$ & $*$ & & & $*$ \\
\hline
\end{tabular}

Note: Base sizes too small to report percentages on Virtual or remote volunteering.

\begin{tabular}{|l|c|c|c|c|c|}
\hline $\begin{array}{l}\text { Other NH } \\
\text { 05/29-06/09/2020 }\end{array}$ & $\begin{array}{c}\text { I anticipate doing } \\
\text { more when } \\
\text { restrictions are } \\
\text { lifted }\end{array}$ & $\begin{array}{c}\text { I anticipate doing } \\
\text { less when } \\
\text { restrictions are } \\
\text { lifted }\end{array}$ & $\begin{array}{c}\text { I anticipate doing } \\
\text { the same amount } \\
\text { when restrictions } \\
\text { are lifted }\end{array}$ & $\begin{array}{c}\text { DON'T } \\
\text { KNOW/ } \\
\text { SKIPPED ON } \\
\text { WEB/ } \\
\text { REFUSED }\end{array}$ & * \\
\hline Video chats & $*$ & $*$ & 84 & - & $N=23$ \\
\hline Telephone calls & 7 & 9 & 72 & - & $N=40$ \\
\hline Text messages & 10 & 18 & 84 & $*$ & $N=32$ \\
\hline Email exchanges & 5 & 11 & $*$ & $*$ & $N=4$ \\
\hline Letter exchanges & $*$ & $*$ & $*$ & $*$ & $N=13$ \\
\hline Live virtual events & $*$ & $*$ & $*$ & $N=2$ \\
\hline $\begin{array}{l}\text { Virtual or remote } \\
\text { volunteering }\end{array}$ & $*$ & & & $*$ & $*$ \\
\hline
\end{tabular}

Note: Base sizes too small to report percentages for Video chats, Letter exchanges, Live virtual events, and Virtual or remote volunteering 
Q20. How, if at all, have your feelings about using technology to connect with others changed during the coronavirus pandemic? Would you say your feelings now are...?

\begin{tabular}{|l|c|c|c|c|c|}
\hline $\begin{array}{l}\text { NORC } \\
\text { 05/29-06/09/2020 }\end{array}$ & Total & White NH & $\begin{array}{c}\text { African } \\
\text { American } \\
\mathrm{NH}\end{array}$ & Hispanic & Other NH \\
\hline Much more positive & 20 & 15 & 26 & 24 & 17 \\
\hline Somewhat more positive & 27 & 30 & 23 & 27 & 17 \\
\hline Not changed & 50 & 52 & 45 & 46 & 59 \\
\hline Somewhat more negative & 2 & 2 & 1 & 1 & 7 \\
\hline Much more negative & 1 & 1 & 2 & 1 & - \\
\hline DON'T KNOW/ SKIPPED ON WEB/ REFUSED & 1 & $*$ & 1 & 1 & - \\
\hline
\end{tabular}

Q21. Have you helped a friend, family member, or neighbor during the coronavirus pandemic?

This could include things like going shopping for them, sending supplies to them, helping them use technology, providing childcare for them, cooking for them, providing financial support to them, moving in together, etc...

\begin{tabular}{|l|c|c|c|c|c|}
\hline $\begin{array}{l}\text { NORC } \\
\text { 05/29-06/09/2020 }\end{array}$ & Total & White NH & $\begin{array}{c}\text { African } \\
\text { American } \\
\mathrm{NH}\end{array}$ & Hispanic & Other NH \\
\hline I have helped someone younger than me & 16 & 16 & 16 & 15 & 28 \\
\hline I have helped someone older than me & 25 & 23 & 26 & 30 & 17 \\
\hline $\begin{array}{l}\text { I have helped someone younger and } \\
\text { someone older than me }\end{array}$ & 25 & 28 & 28 & 18 & 29 \\
\hline I have not helped anyone & 33 & 33 & 30 & 37 & 26 \\
\hline DON'T KNOW/ SKIPPED ON WEB/ REFUSED & $*$ & $*$ & 1 & $*$ & - \\
\hline
\end{tabular}

Q22. Have you received help from a friend, family member, or neighbor during the coronavirus pandemic?

\begin{tabular}{|l|c|c|c|c|c|}
\hline $\begin{array}{l}\text { NORC } \\
\text { 05/29-06/09/2020 }\end{array}$ & Total & White NH & $\begin{array}{c}\text { African } \\
\text { American } \\
\mathrm{NH}\end{array}$ & Hispanic & Other NH \\
\hline $\begin{array}{l}\text { I have gotten help from someone younger } \\
\text { than me }\end{array}$ & 22 & 25 & 22 & 18 & 17 \\
\hline $\begin{array}{l}\text { I have gotten help from someone older than } \\
\text { me }\end{array}$ & 8 & 4 & 12 & 11 & 8 \\
\hline $\begin{array}{l}\text { I have gotten help from someone younger } \\
\text { and someone older than me }\end{array}$ & 14 & 12 & 20 & 11 & 13 \\
\hline I have not gotten help from anyone & 57 & 60 & 45 & 60 & 62 \\
\hline DON'T KNOW/ SKIPPED ON WEB/ REFUSED & $*$ & $*$ & 1 & $*$ & - \\
\hline
\end{tabular}


AARP 50+ Coronavirus Tracker: Wave 5 Oversample

Q23. Do you have parents who are sheltering in place somewhere other than with you?

\begin{tabular}{|l|c|c|c|c|c|}
\hline $\begin{array}{l}\text { NORC } \\
\text { O5/29-06/09/2020 }\end{array}$ & Total & White NH & $\begin{array}{c}\text { African } \\
\text { American NH }\end{array}$ & Hispanic & Other NH \\
\hline Yes & 30 & 29 & 23 & 36 & 38 \\
\hline No & 69 & 70 & 75 & 63 & 62 \\
\hline $\begin{array}{l}\text { DON'T KNOW/ SKIPPED } \\
\text { ON WEB/ REFUSED }\end{array}$ & 1 & 1 & 2 & 1 & 1 \\
\hline
\end{tabular}

Has parents sheltering in place somewhere other than with them

Q24. How worried, if at all, are you about your parents...

[ITEMS RANDOMIZED]

\begin{tabular}{|l|c|c|c|c|c|}
\hline $\begin{array}{l}\text { Total } \\
\text { 05/29-06/09/2020 }\end{array}$ & Very worried & $\begin{array}{c}\text { Somewhat } \\
\text { worried }\end{array}$ & Not too worried & Not at all worried & $\begin{array}{c}\text { DON'T KNOW/ } \\
\text { SKIPPED ON WEB/ } \\
\text { REFUSED }\end{array}$ \\
\hline Staying healthy & 35 & 34 & 21 & 10 & - \\
\hline Feeling lonely & 24 & 32 & 28 & 16 & - \\
\hline
\end{tabular}

$N=570$

\begin{tabular}{|l|c|c|c|c|c|}
\hline $\begin{array}{l}\text { White } \mathrm{NH} \\
\text { 05/29-06/09/2020 }\end{array}$ & Very worried & $\begin{array}{c}\text { Somewhat } \\
\text { worried }\end{array}$ & Not too worried & Not at all worried & $\begin{array}{c}\text { DON'T KNOW/ } \\
\text { SKIPPED ON WEB/ } \\
\text { REFUSED }\end{array}$ \\
\hline Staying healthy & 31 & 40 & 22 & 7 & - \\
\hline Feeling lonely & 26 & 30 & 32 & 11 & - \\
\hline
\end{tabular}

$N=282$

\begin{tabular}{|l|c|c|c|c|c|}
\hline $\begin{array}{l}\text { African American } \mathrm{NH} \\
\text { 05/29-06/09/2020 }\end{array}$ & Very worried & $\begin{array}{c}\text { Somewhat } \\
\text { worried }\end{array}$ & Not too worried & Not at all worried & $\begin{array}{c}\text { DON'T KNOW/ } \\
\text { SKIPPED ON WEB/ } \\
\text { REFUSED }\end{array}$ \\
\hline Staying healthy & 36 & 31 & 22 & 11 & - \\
\hline Feeling lonely & 22 & 31 & 17 & 30 & - \\
\hline
\end{tabular}

$N=102$

\begin{tabular}{|l|c|c|c|c|c|}
\hline $\begin{array}{l}\text { Hispanic } \\
\text { 05/29-06/09/2020 }\end{array}$ & Very worried & $\begin{array}{c}\text { Somewhat } \\
\text { worried }\end{array}$ & Not too worried & Not at all worried & $\begin{array}{c}\text { DON'T KNOW/ } \\
\text { SKIPPED ON WEB/ } \\
\text { REFUSED }\end{array}$ \\
\hline Staying healthy & 43 & 28 & 20 & 10 & - \\
\hline Feeling lonely & 24 & 32 & 32 & 12 & - \\
\hline
\end{tabular}

$N=169$ 


\begin{tabular}{|l|c|c|c|c|c|}
\hline $\begin{array}{l}\text { Other } \mathrm{NH} \\
\text { 05/29-06/09/2020 }\end{array}$ & $\begin{array}{c}\text { Somewhat } \\
\text { worried }\end{array}$ & Not too worried & Not at all worried & $\begin{array}{c}\text { DON'T KNOW/ } \\
\text { SKIPPED ON WEB/ } \\
\text { REFUSED }\end{array}$ \\
\hline Staying healthy & 30 & 30 & 8 & 32 & - \\
\hline Feeling lonely & 9 & 44 & 12 & 35 & - \\
\hline
\end{tabular}

Note: Base sizes too small to report percentages.

Q25. What is your level of agreement/disagreement with the following statement: When coronavirus restrictions are lifted, older Americans should continue social distancing longer than younger Americans for their own health and safety.

\begin{tabular}{|l|c|c|c|c|c|}
\hline $\begin{array}{l}\text { NORC } \\
\text { 05/29-06/09/2020 }\end{array}$ & Total & White NH & $\begin{array}{c}\text { African } \\
\text { American } \\
\mathrm{NH}\end{array}$ & Hispanic & Other NH \\
\hline Strongly agree & 47 & 43 & 55 & 50 & 41 \\
\hline Somewhat agree & 38 & 44 & 36 & 31 & 28 \\
\hline Somewhat disagree & 8 & 8 & 5 & 10 & 19 \\
\hline Strongly disagree & 5 & 5 & 3 & 7 & 5 \\
\hline DON'T KNOW/ SKIPPED ON WEB/ REFUSED & 1 & $*$ & 1 & 2 & 6 \\
\hline
\end{tabular}

Q26. When social distancing restrictions are lifted, do you plan to be among the first, middle, or last group of people to attend in-person social gatherings?

\begin{tabular}{|l|c|c|c|c|c|}
\hline $\begin{array}{l}\text { NORC } \\
\text { 05/29-06/09/2020 }\end{array}$ & Total & White NH & $\begin{array}{c}\text { African } \\
\text { American } \\
\mathrm{NH}\end{array}$ & Hispanic & Other $\mathrm{NH}$ \\
\hline First & 11 & 12 & 6 & 10 & 20 \\
\hline Middle & 37 & 41 & 29 & 38 & 32 \\
\hline Last & 51 & 46 & 64 & 51 & 44 \\
\hline DON'T KNOW/ SKIPPED ON WEB/ REFUSED & 1 & 1 & 1 & 1 & 5 \\
\hline
\end{tabular}


Q27. What types of fun, virtual events have you participated in since the onset of the coronavirus pandemic requiring most people to shelter in place?

Please select all that apply.

\section{[ITEMS RANDOMIZED]}

\begin{tabular}{|c|c|c|c|c|c|}
\hline $\begin{array}{l}\text { NORC } \\
05 / 29-06 / 09 / 2020\end{array}$ & Total & White NH & $\begin{array}{c}\text { African } \\
\text { American } \\
\mathrm{NH}\end{array}$ & Hispanic & Other $\mathrm{NH}$ \\
\hline Music events (e.g. virtual concert) & 12 & 14 & 12 & 7 & 11 \\
\hline Dance parties (e.g. Daybreakers, DJ D-Nice) & 3 & 2 & 4 & 4 & 1 \\
\hline Food \& Beverage events (e.g. live cooking class) & 6 & 4 & 6 & 8 & 1 \\
\hline Movie/Theatre events (e.g. Watching Party) & 8 & 7 & 13 & 5 & 7 \\
\hline $\begin{array}{l}\text { Exercise (e.g. yoga class, dance class, gym workout } \\
\text { class) }\end{array}$ & 17 & 14 & 20 & 19 & 13 \\
\hline $\begin{array}{l}\text { Educational events/lectures (e.g. MasterClass, } \\
\text { Great Course) }\end{array}$ & 11 & 11 & 13 & 9 & 14 \\
\hline Personal sport competitions (e.g. virtual 5k) & 3 & 2 & 5 & 3 & 5 \\
\hline Museum events (e.g. virtual tour) & 5 & 6 & 3 & 5 & 9 \\
\hline Library events (e.g. book launch, reading) & 6 & 6 & 7 & 5 & 8 \\
\hline Travel events (e.g. virtual tour) & 7 & 6 & 6 & 7 & 14 \\
\hline $\begin{array}{l}\text { Gaming events (e.g. trivia night, card game } \\
\text { tournament) }\end{array}$ & 9 & 9 & 11 & 9 & 9 \\
\hline Virtual volunteering & 5 & 6 & 4 & 4 & 7 \\
\hline None of the above & 59 & 58 & 57 & 61 & 59 \\
\hline DON'T KNOW/ SKIPPED ON WEB/ REFUSED & 1 & $*$ & 1 & 1 & - \\
\hline
\end{tabular}


Q28. Has the coronavirus impacted your travel plans in any of the following ways?

[ITEMS RANDOMIZED]

\begin{tabular}{|c|c|c|c|}
\hline $\begin{array}{l}\text { Total } \\
05 / 29-06 / 09 / 2020\end{array}$ & Yes & No & $\begin{array}{l}\text { DON'T } \\
\text { KNOW/ } \\
\text { SKIPPED } \\
\text { ON WEB/ } \\
\text { REFUSED }\end{array}$ \\
\hline $\begin{array}{l}\text { I have postponed or cancelled leisure travel plans to protect my health (or } \\
\text { the health of loved ones) due to coronavirus }\end{array}$ & 60 & 40 & 1 \\
\hline $\begin{array}{l}\text { I have postponed or cancelled leisure travel plans to save money due to } \\
\text { coronavirus }\end{array}$ & 32 & 67 & 1 \\
\hline $\begin{array}{l}\text { I have changed the destination of leisure travel plans to avoid areas affected } \\
\text { by coronavirus }\end{array}$ & 41 & 57 & 2 \\
\hline I have postponed or cancelled cruise plans due to coronavirus & 23 & 75 & 2 \\
\hline $\begin{array}{l}\text { I have changed the type of transportation (ex. flight, train, car) I will take to } \\
\text { arrive at my leisure travel destination due to coronavirus }\end{array}$ & 35 & 64 & 1 \\
\hline $\begin{array}{l}\text { I have changed who I will travel with so it is limited to people I quarantined } \\
\text { with due to coronavirus }\end{array}$ & 30 & 69 & 1 \\
\hline I will avoid leisure travel until there is a coronavirus vaccine & 51 & 48 & 2 \\
\hline
\end{tabular}

$N=1,798$

White NH

05/29-06/09/2020

I have postponed or cancelled leisure travel plans to protect my health (or the health of loved ones) due to coronavirus

I have postponed or cancelled leisure travel plans to save money due to coronavirus

I have changed the destination of leisure travel plans to avoid areas affected by coronavirus

I have postponed or cancelled cruise plans due to coronavirus

I have changed the type of transportation (ex. flight, train, car) I will take to arrive at my leisure travel destination due to coronavirus

I have changed who I will travel with so it is limited to people I quarantined with due to coronavirus

I will avoid leisure travel until there is a coronavirus vaccine

$N=906$ 


\begin{tabular}{|c|c|c|c|}
\hline $\begin{array}{l}\text { African American NH } \\
05 / 29-06 / 09 / 2020\end{array}$ & Yes & No & $\begin{array}{c}\text { DON'T } \\
\text { KNOW/ } \\
\text { SKIPPED } \\
\text { ON WEB/ } \\
\text { REFUSED }\end{array}$ \\
\hline $\begin{array}{l}\text { I have postponed or cancelled leisure travel plans to protect my health (or } \\
\text { the health of loved ones) due to coronavirus }\end{array}$ & 66 & 33 & 1 \\
\hline $\begin{array}{l}\text { I have postponed or cancelled leisure travel plans to save money due to } \\
\text { coronavirus }\end{array}$ & 44 & 55 & 1 \\
\hline $\begin{array}{l}\text { I have changed the destination of leisure travel plans to avoid areas affected } \\
\text { by coronavirus }\end{array}$ & 52 & 47 & 1 \\
\hline I have postponed or cancelled cruise plans due to coronavirus & 40 & 59 & 2 \\
\hline $\begin{array}{l}\text { I have changed the type of transportation (ex. flight, train, car) I will take to } \\
\text { arrive at my leisure travel destination due to coronavirus }\end{array}$ & 47 & 52 & 1 \\
\hline $\begin{array}{l}\text { I have changed who I will travel with so it is limited to people I quarantined } \\
\text { with due to coronavirus }\end{array}$ & 43 & 56 & 2 \\
\hline I will avoid leisure travel until there is a coronavirus vaccine & 65 & 33 & 3 \\
\hline
\end{tabular}

$N=401$

\begin{tabular}{|c|c|c|c|}
\hline $\begin{array}{l}\text { Hispanic } \\
05 / 29-06 / 09 / 2020\end{array}$ & Yes & No & $\begin{array}{c}\text { DON'T } \\
\text { KNOW/ } \\
\text { SKIPPED } \\
\text { ON WEB/ } \\
\text { REFUSED }\end{array}$ \\
\hline $\begin{array}{l}\text { I have postponed or cancelled leisure travel plans to protect my health (or } \\
\text { the health of loved ones) due to coronavirus }\end{array}$ & 60 & 39 & 1 \\
\hline $\begin{array}{l}\text { I have postponed or cancelled leisure travel plans to save money due to } \\
\text { coronavirus }\end{array}$ & 37 & 62 & 1 \\
\hline $\begin{array}{l}\text { I have changed the destination of leisure travel plans to avoid areas affected } \\
\text { by coronavirus }\end{array}$ & 47 & 52 & 1 \\
\hline I have postponed or cancelled cruise plans due to coronavirus & 24 & 76 & 1 \\
\hline $\begin{array}{l}\text { I have changed the type of transportation (ex. flight, train, car) I will take to } \\
\text { arrive at my leisure travel destination due to coronavirus }\end{array}$ & 38 & 61 & 1 \\
\hline $\begin{array}{l}\text { I have changed who I will travel with so it is limited to people I quarantined } \\
\text { with due to coronavirus }\end{array}$ & 37 & 62 & 1 \\
\hline I will avoid leisure travel until there is a coronavirus vaccine & 51 & 47 & 2 \\
\hline
\end{tabular}




\begin{tabular}{|c|c|c|c|}
\hline $\begin{array}{l}\text { Other } \mathrm{NH} \\
05 / 29-06 / 09 / 2020\end{array}$ & Yes & No & $\begin{array}{c}\text { DON'T } \\
\text { KNOW/ } \\
\text { SKIPPED } \\
\text { ON WEB/ } \\
\text { REFUSED }\end{array}$ \\
\hline $\begin{array}{l}\text { I have postponed or cancelled leisure travel plans to protect my health (or } \\
\text { the health of loved ones) due to coronavirus }\end{array}$ & 57 & 43 & - \\
\hline $\begin{array}{l}\text { I have postponed or cancelled leisure travel plans to save money due to } \\
\text { coronavirus }\end{array}$ & 36 & 63 & 1 \\
\hline $\begin{array}{l}\text { I have changed the destination of leisure travel plans to avoid areas affected } \\
\text { by coronavirus }\end{array}$ & 34 & 60 & 6 \\
\hline I have postponed or cancelled cruise plans due to coronavirus & 18 & 77 & 6 \\
\hline $\begin{array}{l}\text { I have changed the type of transportation (ex. flight, train, car) I will take to } \\
\text { arrive at my leisure travel destination due to coronavirus }\end{array}$ & 35 & 65 & - \\
\hline $\begin{array}{l}\text { I have changed who I will travel with so it is limited to people I quarantined } \\
\text { with due to coronavirus }\end{array}$ & 23 & 76 & 1 \\
\hline I will avoid leisure travel until there is a coronavirus vaccine & 52 & 48 & - \\
\hline
\end{tabular}

Q29. When you think about spending time with others in person after the pandemic, what are you most looking forward to?

Please select up to three of the following.

[ITEMS RANDOMIZED, SELECT UP TO THREE]

\begin{tabular}{|l|c|c|c|c|c|}
\hline $\begin{array}{l}\text { NORC } \\
\text { 05/29-06/09/2020 }\end{array}$ & Total & White NH & $\begin{array}{c}\text { African } \\
\text { American } \\
\mathrm{NH}\end{array}$ & Hispanic & Other NH \\
\hline Having meals or drinks together & 57 & 65 & 46 & 50 & 69 \\
\hline $\begin{array}{l}\text { Celebrating milestones together (e.g., birthdays, } \\
\text { graduations, reunions, etc.) }\end{array}$ & 41 & 45 & 33 & 40 & 36 \\
\hline Hugging people I love & 48 & 52 & 41 & 45 & 52 \\
\hline Exercising or playing sports together & 14 & 13 & 13 & 17 & 10 \\
\hline Just hanging out together & 49 & 51 & 49 & 47 & 47 \\
\hline Going shopping together & 16 & 15 & 19 & 18 & 9 \\
\hline Traveling together & 20 & 18 & 22 & 27 & 5 \\
\hline Playing games together & 10 & 9 & 14 & 9 & 13 \\
\hline Other (specify): & 3 & 3 & 4 & 4 & 4 \\
\hline None of the above & 3 & 2 & 6 & 1 & 7 \\
\hline DON'T KNOW/ SKIPPED ON WEB/ REFUSED & $*$ & $*$ & 1 & 1 & - \\
\hline
\end{tabular}


Q30. What are the greatest benefits of social connections for you?

Please select up to three of the following.

[ITEMS RANDOMIZED, SELECT UP TO THREE]

\begin{tabular}{|l|c|c|c|c|c|}
\hline NORC & Total & White NH & $\begin{array}{c}\text { African } \\
\text { American } \\
\text { NH }\end{array}$ & Hispanic & Other NH \\
\hline Having a sense of belonging & 17 & 23 & 10 & 11 & 20 \\
\hline Providing a sense of purpose/meaning & 14 & 15 & 12 & 15 & 12 \\
\hline Making you feel valued & 7 & 7 & 6 & 8 & 6 \\
\hline Increasing your happiness & 22 & 27 & 15 & 18 & 23 \\
\hline Decreasing your stress & 13 & 11 & 14 & 16 & 12 \\
\hline Easing your feelings of loneliness and isolation & 14 & 17 & 11 & 11 & 23 \\
\hline Helping you cope with life events & 8 & 8 & 10 & 8 & 6 \\
\hline Having someone to do activities with & 25 & 30 & 19 & 22 & 21 \\
\hline Helping you solve problems & 4 & 4 & 3 & 4 & 9 \\
\hline Helping you maintain a healthy lifestyle & 19 & 14 & 24 & 27 & 10 \\
\hline Having someone to talk to & 27 & 28 & 24 & 26 & 32 \\
\hline Meeting people & 14 & 13 & 17 & 12 & 15 \\
\hline Feeling welcome & 11 & 11 & 12 & 10 & 2 \\
\hline Having fun & 37 & 44 & 31 & 30 & 34 \\
\hline Learning new things & 13 & 11 & 15 & 15 & 11 \\
\hline Experiencing growth & 7 & 4 & 12 & 7 & 9 \\
\hline Seeing new possibilities & 5 & 4 & 9 & 5 & 3 \\
\hline Being energized/supported & 11 & 11 & 13 & 11 & 12 \\
\hline DON'T KNOW/ SKIPPED ON WEB/ REFUSED & 3 & 1 & 6 & 5 & 3 \\
\hline
\end{tabular}

Q31. Has your sex life gotten better, worse, or stayed the same during the coronavirus pandemic?

\begin{tabular}{|l|c|c|c|c|c|}
\hline $\begin{array}{l}\text { NORC } \\
\text { 05/29-06/09/2020 }\end{array}$ & Total & White NH & $\begin{array}{c}\text { African } \\
\text { American } \\
\mathrm{NH}\end{array}$ & Hispanic & Other NH \\
\hline Better & 5 & 4 & 7 & 8 & 5 \\
\hline Worse & 10 & 9 & 12 & 10 & 11 \\
\hline Stayed the same & 82 & 86 & 77 & 78 & 80 \\
\hline DON'T KNOW/ SKIPPED ON WEB/ REFUSED & 3 & 2 & 4 & 5 & 4 \\
\hline
\end{tabular}


Q32. Do you believe there have been any of the following positive outcomes from the coronavirus pandemic?

Please select all that apply.

[ITEMS RANDOMIZED]

\begin{tabular}{|l|c|c|c|c|c|}
\hline $\begin{array}{l}\text { NORC } \\
\text { 05/29-06/09/2020 }\end{array}$ & Total & White NH & $\begin{array}{c}\text { African } \\
\text { American } \\
\text { NH }\end{array}$ & Hispanic & Other NH \\
\hline My personal wellbeing or wellness has improved & 18 & 10 & 30 & 20 & 18 \\
\hline I feel more connected to others & 17 & 10 & 26 & 23 & 14 \\
\hline I used a new technology & 24 & 26 & 30 & 17 & 21 \\
\hline I received unexpected support from others & 15 & 11 & 25 & 15 & 19 \\
\hline I have grown closer to family & 33 & 25 & 44 & 39 & 22 \\
\hline $\begin{array}{l}\text { Collectively, people have reduced their impact } \\
\text { on the environment }\end{array}$ & 39 & 45 & 32 & 34 & 38 \\
\hline I have taken more time for myself & 40 & 33 & 51 & 44 & 26 \\
\hline I learned something new & 30 & 24 & 42 & 29 & 28 \\
\hline I got to help someone in need & 26 & 20 & 36 & 27 & 24 \\
\hline Other (specify): & 5 & 5 & 4 & 4 & 13 \\
\hline None of the above & 14 & 16 & 9 & 12 & 25 \\
\hline DON'T KNOW/ SKIPPED ON WEB/ REFUSED & $*$ & $*$ & 1 & $*$ & - \\
\hline
\end{tabular}

Q33. Are you currently sheltering in place due to the coronavirus pandemic?

\begin{tabular}{|l|c|c|c|c|c|}
\hline $\begin{array}{l}\text { NORC } \\
05 / 29-06 / 09 / 2020\end{array}$ & Total & White NH & $\begin{array}{c}\text { African } \\
\text { American NH }\end{array}$ & Hispanic & Other NH \\
\hline Yes & 59 & 59 & 66 & 52 & 56 \\
\hline No & 41 & 40 & 33 & 47 & 43 \\
\hline $\begin{array}{l}\text { DON'T KNOW/ SKIPPED } \\
\text { ON WEB/ REFUSED }\end{array}$ & 1 & 1 & 1 & 1 & 1 \\
\hline
\end{tabular}


AARP 50+ Coronavirus Tracker: Wave 5 Oversample

If sheltering in place

Q34. Whom are you sheltering in place with?

[ITEMS RANDOMIZED]

\begin{tabular}{|c|c|c|c|c|c|}
\hline $\begin{array}{l}\text { NORC } \\
05 / 29-06 / 09 / 2020\end{array}$ & Total & White $\mathrm{NH}$ & $\begin{array}{c}\text { African } \\
\text { American } \\
\mathrm{NH}\end{array}$ & Hispanic & Other $\mathrm{NH}$ \\
\hline Children who are under 18 years old & 10 & 6 & 11 & 17 & $*$ \\
\hline Children who are over 18 years old & 18 & 16 & 22 & 21 & $*$ \\
\hline Spouse, life partner, or significant other & 50 & 62 & 32 & 43 & $*$ \\
\hline Parent & 6 & 3 & 4 & 16 & $*$ \\
\hline Grandparent & $*$ & $*$ & $*$ & - & $*$ \\
\hline $\begin{array}{l}\text { Family member other than child, parent, } \\
\text { grandparent, spouse }\end{array}$ & 7 & 4 & 12 & 8 & $*$ \\
\hline Friend & 3 & 2 & 2 & 5 & $*$ \\
\hline Other (specify): & 5 & 2 & 6 & 12 & $*$ \\
\hline No one. I'm sheltering in place alone & 27 & 26 & 31 & 21 & $*$ \\
\hline DON'T KNOW/ SKIPPED ON WEB/ REFUSED & $*$ & - & $*$ & - & $*$ \\
\hline
\end{tabular}

Note: Base sizes too small to report percentages for Other NH. 
AARP 50+ Coronavirus Tracker: Wave 5 Oversample

If any people not selected as sheltering in place with Q35. How often are you communicating with the following people you are not sheltering in place with?

[ITEMS RANDOMIZED]

\begin{tabular}{|c|c|c|c|c|c|c|c|c|c|}
\hline $\begin{array}{l}\text { Total } \\
05 / 29-06 / 09 / 2020\end{array}$ & $\begin{array}{l}\text { Multiple } \\
\text { times } \\
\text { per day }\end{array}$ & $\begin{array}{c}\text { Once a } \\
\text { day }\end{array}$ & $\begin{array}{c}\text { A few } \\
\text { times a } \\
\text { week }\end{array}$ & $\begin{array}{c}\text { Once per } \\
\text { week }\end{array}$ & $\begin{array}{c}\text { Once } \\
\text { every } \\
\text { few } \\
\text { weeks }\end{array}$ & $\begin{array}{l}\text { Once a } \\
\text { month or } \\
\text { less }\end{array}$ & $\begin{array}{l}\text { Don't } \\
\text { have this } \\
\text { type of } \\
\text { person in } \\
\text { my life }\end{array}$ & $\begin{array}{l}\text { DON'T } \\
\text { KNOW/ } \\
\text { SKIPPED } \\
\text { ON WEB/ } \\
\text { REFUSED }\end{array}$ & \\
\hline $\begin{array}{l}\text { Children who are } \\
\text { under } 18 \text { years old }\end{array}$ & 4 & 2 & 9 & 7 & 5 & 5 & 67 & 2 & $N=991$ \\
\hline $\begin{array}{l}\text { Children who are } \\
\text { over } 18 \text { years old }\end{array}$ & 13 & 11 & 26 & 13 & 6 & 5 & 27 & $*$ & $N=884$ \\
\hline $\begin{array}{l}\text { Spouse, life } \\
\text { partner, or } \\
\text { significant other }\end{array}$ & 12 & 6 & 4 & 2 & 1 & 1 & 74 & 1 & $N=522$ \\
\hline Parent & 5 & 7 & 10 & 6 & 3 & 4 & 63 & 2 & $N=1,060$ \\
\hline Grandparent & 1 & 1 & 1 & 2 & 1 & 1 & 93 & 1 & $N=1,095$ \\
\hline $\begin{array}{l}\text { Family member } \\
\text { other than child, } \\
\text { parent, } \\
\text { grandparent, } \\
\text { spouse }\end{array}$ & 10 & 11 & 27 & 14 & 13 & 9 & 17 & 1 & $N=1,031$ \\
\hline Friend & 12 & 10 & 36 & 15 & 10 & 9 & 5 & 2 & $N=1,071$ \\
\hline
\end{tabular}




\begin{tabular}{|c|c|c|c|c|c|c|c|c|c|}
\hline $\begin{array}{l}\text { White NH } \\
05 / 29-06 / 09 / 2020\end{array}$ & $\begin{array}{l}\text { Multiple } \\
\text { times } \\
\text { per day }\end{array}$ & $\begin{array}{c}\text { Once a } \\
\text { day }\end{array}$ & $\begin{array}{c}\text { A few } \\
\text { times a } \\
\text { week }\end{array}$ & $\begin{array}{c}\text { Once } \\
\text { per } \\
\text { week }\end{array}$ & $\begin{array}{c}\text { Once } \\
\text { every few } \\
\text { weeks }\end{array}$ & $\begin{array}{l}\text { Once a } \\
\text { month or } \\
\text { less }\end{array}$ & $\begin{array}{l}\text { Don't } \\
\text { have this } \\
\text { type of } \\
\text { person in } \\
\text { my life }\end{array}$ & $\begin{array}{l}\text { DON'T } \\
\text { KNOW/ } \\
\text { SKIPPED } \\
\text { ON WEB/ } \\
\text { REFUSED }\end{array}$ & \\
\hline $\begin{array}{l}\text { Children who are } \\
\text { under } 18 \text { years old }\end{array}$ & 1 & 2 & 9 & 6 & 6 & 2 & 74 & 1 & $N=508$ \\
\hline $\begin{array}{l}\text { Children who are } \\
\text { over } 18 \text { years old }\end{array}$ & 8 & 12 & 30 & 12 & 6 & 4 & 27 & 1 & $N=461$ \\
\hline $\begin{array}{l}\text { Spouse, life } \\
\text { partner, or } \\
\text { significant other }\end{array}$ & 5 & 8 & 6 & $*$ & 1 & $*$ & 80 & $*$ & $N=206$ \\
\hline Parent & 4 & 6 & 10 & 5 & 4 & 3 & 67 & 2 & $N=533$ \\
\hline Grandparent & $*$ & - & $*$ & 1 & 1 & 1 & 96 & 1 & $N=541$ \\
\hline $\begin{array}{l}\text { Family member } \\
\text { other than child, } \\
\text { parent, } \\
\text { grandparent, } \\
\text { spouse }\end{array}$ & 5 & 8 & 25 & 19 & 18 & 8 & 17 & 1 & $N=520$ \\
\hline Friend & 13 & 9 & 37 & 16 & 10 & 9 & 5 & 2 & $N=533$ \\
\hline
\end{tabular}

\begin{tabular}{|c|c|c|c|c|c|c|c|c|c|}
\hline $\begin{array}{l}\text { African American } \\
\mathrm{NH} \\
05 / 29-06 / 09 / 2020\end{array}$ & $\begin{array}{l}\text { Multiple } \\
\text { times } \\
\text { per day }\end{array}$ & $\begin{array}{c}\text { Once a } \\
\text { day }\end{array}$ & $\begin{array}{l}\text { A few } \\
\text { times a } \\
\text { week }\end{array}$ & $\begin{array}{l}\text { Once } \\
\text { per } \\
\text { week }\end{array}$ & $\begin{array}{l}\text { Once } \\
\text { every few } \\
\text { weeks }\end{array}$ & $\begin{array}{l}\text { Once a } \\
\text { month or } \\
\text { less }\end{array}$ & $\begin{array}{l}\text { Don't } \\
\text { have this } \\
\text { type of } \\
\text { person in } \\
\text { my life }\end{array}$ & $\begin{array}{l}\text { DON'T } \\
\text { KNOW/ } \\
\text { SKIPPED } \\
\text { ON WEB/ } \\
\text { REFUSED }\end{array}$ & \\
\hline $\begin{array}{l}\text { Children who are } \\
\text { under } 18 \text { years old }\end{array}$ & 8 & 5 & 13 & 6 & 3 & 4 & 57 & 3 & $N=245$ \\
\hline $\begin{array}{l}\text { Children who are } \\
\text { over } 18 \text { years old }\end{array}$ & 17 & 13 & 23 & 8 & 6 & 7 & 25 & * & $N=212$ \\
\hline $\begin{array}{l}\text { Spouse, life } \\
\text { partner, or } \\
\text { significant other }\end{array}$ & 20 & 4 & 4 & 3 & 1 & 1 & 64 & 2 & $N=185$ \\
\hline Parent & 7 & 10 & 10 & 4 & 3 & 4 & 60 & 3 & $N=266$ \\
\hline Grandparent & - & 1 & 2 & 4 & $*$ & 1 & 89 & 3 & $N=276$ \\
\hline $\begin{array}{l}\text { Family member } \\
\text { other than child, } \\
\text { parent, } \\
\text { grandparent, } \\
\text { spouse }\end{array}$ & 18 & 10 & 30 & 8 & 7 & 10 & 15 & 1 & $N=253$ \\
\hline Friend & 16 & 14 & 33 & 12 & 8 & 9 & 6 & 2 & $N=267$ \\
\hline
\end{tabular}




\begin{tabular}{|c|c|c|c|c|c|c|c|c|c|}
\hline $\begin{array}{l}\text { Hispanic } \\
05 / 29-06 / 09 / 2020\end{array}$ & $\begin{array}{c}\text { Multiple } \\
\text { times } \\
\text { per day }\end{array}$ & $\begin{array}{c}\text { Once a } \\
\text { day }\end{array}$ & $\begin{array}{c}\text { A few } \\
\text { times a } \\
\text { week }\end{array}$ & $\begin{array}{c}\text { Once } \\
\text { per } \\
\text { week }\end{array}$ & $\begin{array}{c}\text { Once } \\
\text { every few } \\
\text { weeks }\end{array}$ & $\begin{array}{c}\text { Once a } \\
\text { month or } \\
\text { less }\end{array}$ & $\begin{array}{l}\text { Don't have } \\
\text { this type of } \\
\text { person in } \\
\text { my life }\end{array}$ & $\begin{array}{l}\text { DON'T } \\
\text { KNOW/ } \\
\text { SKIPPED } \\
\text { ON WEB/ } \\
\text { REFUSED }\end{array}$ & \\
\hline $\begin{array}{l}\text { Children who are } \\
\text { under } 18 \text { years old }\end{array}$ & 5 & 1 & 4 & 10 & 5 & 15 & 59 & 1 & $N=212$ \\
\hline $\begin{array}{l}\text { Children who are } \\
\text { over } 18 \text { years old }\end{array}$ & 21 & 4 & 19 & 19 & 6 & 4 & 27 & $*$ & $N=187$ \\
\hline $\begin{array}{l}\text { Spouse, life } \\
\text { partner, or } \\
\text { significant other }\end{array}$ & 9 & 9 & 2 & 3 & $*$ & $*$ & 76 & 1 & $N=118$ \\
\hline Parent & 9 & 6 & 7 & 10 & 3 & 5 & 58 & 1 & $N=232$ \\
\hline Grandparent & 4 & 3 & 1 & 1 & 1 & 4 & 88 & $*$ & $N=249$ \\
\hline $\begin{array}{l}\text { Family member } \\
\text { other than child, } \\
\text { parent, } \\
\text { grandparent, } \\
\text { spouse }\end{array}$ & 12 & 20 & 26 & 11 & 5 & 9 & 16 & 1 & $N=231$ \\
\hline Friend & 8 & 10 & 41 & 17 & 14 & 7 & 4 & 1 & $N=243$ \\
\hline
\end{tabular}

\begin{tabular}{|c|c|c|c|c|c|c|c|c|c|}
\hline $\begin{array}{l}\text { Other NH } \\
05 / 29-06 / 09 / 2020\end{array}$ & $\begin{array}{l}\text { Multiple } \\
\text { times } \\
\text { per day }\end{array}$ & $\begin{array}{c}\text { Once a } \\
\text { day }\end{array}$ & $\begin{array}{c}\text { A few } \\
\text { times a } \\
\text { week }\end{array}$ & $\begin{array}{c}\text { Once } \\
\text { per } \\
\text { week }\end{array}$ & $\begin{array}{l}\text { Once } \\
\text { every few } \\
\text { weeks }\end{array}$ & $\begin{array}{c}\text { Once a } \\
\text { month or } \\
\text { less }\end{array}$ & $\begin{array}{l}\text { Don't have } \\
\text { this type of } \\
\text { person in } \\
\text { my life }\end{array}$ & $\begin{array}{l}\text { DON'T } \\
\text { KNOW/ } \\
\text { SKIPPED } \\
\text { ON WEB/ } \\
\text { REFUSED }\end{array}$ & \\
\hline $\begin{array}{l}\text { Children who are } \\
\text { under } 18 \text { years old }\end{array}$ & $*$ & $*$ & $*$ & $*$ & $*$ & $*$ & $*$ & $*$ & $N=26$ \\
\hline $\begin{array}{l}\text { Children who are } \\
\text { over } 18 \text { years old }\end{array}$ & $*$ & $*$ & $*$ & $*$ & $*$ & $*$ & $*$ & $*$ & $N=24$ \\
\hline $\begin{array}{l}\text { Spouse, life } \\
\text { partner, or } \\
\text { significant other }\end{array}$ & $*$ & $*$ & $*$ & $*$ & $*$ & $*$ & $*$ & $*$ & $N=13$ \\
\hline Parent & $*$ & $*$ & $*$ & $*$ & $*$ & $*$ & $*$ & $*$ & $N=29$ \\
\hline Grandparent & $*$ & $*$ & $*$ & $*$ & $*$ & $*$ & $*$ & $*$ & $N=29$ \\
\hline $\begin{array}{l}\text { Family member } \\
\text { other than child, } \\
\text { parent, } \\
\text { grandparent, } \\
\text { spouse }\end{array}$ & $*$ & $*$ & * & $*$ & $*$ & $*$ & $*$ & * & $N=27$ \\
\hline Friend & $*$ & $*$ & $*$ & $*$ & $*$ & $*$ & $*$ & $*$ & $N=28$ \\
\hline
\end{tabular}

Note: Base sizes too small to report percentages. 
AARP 50+ Coronavirus Tracker: Wave 5 Oversample

SURV_LANG. Survey interview language

\begin{tabular}{|l|c|c|c|c|c|}
\hline NORC & Total & White NH & $\begin{array}{c}\text { African } \\
\text { American NH }\end{array}$ & Hispanic & Other NH \\
\hline English & 97 & 100 & 100 & 88 & 100 \\
\hline Spanish & 3 & - & - & 12 & - \\
\hline
\end{tabular}

SURV_MODE. Survey interview mode

\begin{tabular}{|l|c|c|c|c|c|}
\hline NORC & Total & White NH & $\begin{array}{c}\text { African } \\
\text { American NH }\end{array}$ & Hispanic & Other NH \\
\hline Phone & 16 & 6 & 32 & 19 & 23 \\
\hline Online & 84 & 95 & 68 & 81 & 77 \\
\hline
\end{tabular}

DEVICE. Device

\begin{tabular}{|l|c|c|c|c|c|}
\hline $\begin{array}{l}\text { NORC } \\
\text { 05/29-06/09/2020 }\end{array}$ & Total & White NH & $\begin{array}{c}\text { African } \\
\text { American } \\
\mathrm{NH}\end{array}$ & Hispanic & Other $\mathrm{NH}$ \\
\hline Desktop & 43 & 55 & 27 & 31 & 54 \\
\hline $\begin{array}{l}\text { Phone interview (not } \\
\text { online) }\end{array}$ & 16 & 6 & 32 & 19 & 23 \\
\hline Smartphone & 39 & 37 & 40 & 48 & 23 \\
\hline Tablet & 2 & 3 & 2 & 2 & - \\
\hline
\end{tabular}

GENDER. Gender

\begin{tabular}{|l|c|c|c|c|c|}
\hline $\begin{array}{l}\text { NORC } \\
\text { 05/29-06/09/2020 }\end{array}$ & Total & White NH & $\begin{array}{c}\text { African } \\
\text { American NH }\end{array}$ & Hispanic & Other NH \\
\hline Male & 46 & 46 & 43 & 47 & 55 \\
\hline Female & 54 & 54 & 57 & 53 & 45 \\
\hline
\end{tabular}

AGE2. Age -2 categories

\begin{tabular}{|l|c|c|c|c|c|}
\hline NORC & Total & White NH & $\begin{array}{c}\text { African } \\
\text { American NH }\end{array}$ & Hispanic & Other NH \\
\hline $50-64$ & 57 & 52 & 61 & 67 & 50 \\
\hline $65+$ & 43 & 49 & 39 & 33 & 50 \\
\hline
\end{tabular}


AARP 50+ Coronavirus Tracker: Wave 5 Oversample

AGE3. Age -3 categories

\begin{tabular}{|l|c|c|c|c|c|}
\hline $\begin{array}{l}\text { NORC } \\
05 / 29-06 / 09 / 2020\end{array}$ & Total & White NH & $\begin{array}{c}\text { African } \\
\text { American NH }\end{array}$ & Hispanic & Other NH \\
\hline $50-59$ & 39 & 34 & 42 & 47 & 34 \\
\hline $60-69$ & 33 & 34 & 34 & 30 & 30 \\
\hline $70+$ & 29 & 32 & 25 & 23 & 37 \\
\hline
\end{tabular}

RACETHNICITY. Combined race/ethnicity

\begin{tabular}{|l|c|c|c|c|c|}
\hline NORC & Total & White NH & $\begin{array}{c}\text { African } \\
\text { American NH }\end{array}$ & Hispanic & Other NH \\
\hline White, non-Hispanic & 48 & 100 & - & - & - \\
\hline Black, non-Hispanic & 22 & - & 100 & - & - \\
\hline Other, non-Hispanic & 2 & - & - & - & 35 \\
\hline Hispanic & 25 & - & - & 100 & - \\
\hline $2+$, non-Hispanic & 2 & - & - & - & 32 \\
\hline Asian, non-Hispanic & 2 & - & - & - & 33 \\
\hline
\end{tabular}

EDUC. Education (highest degree received)

\begin{tabular}{|l|c|c|c|c|c|}
\hline $\begin{array}{l}\text { NORC } \\
\text { 05/29-06/09/2020 }\end{array}$ & Total & White NH & $\begin{array}{c}\text { African } \\
\text { American } \\
\mathrm{NH}\end{array}$ & Hispanic & Other NH \\
\hline No high school diploma & 16 & 7 & 15 & 35 & 9 \\
\hline $\begin{array}{l}\text { High school graduate or } \\
\text { equivalent }\end{array}$ & 31 & 32 & 35 & 29 & 16 \\
\hline Some college & 25 & 26 & 28 & 18 & 34 \\
\hline Bachelor's degree or above & 15 & 19 & 11 & 10 & 27 \\
\hline Graduate degree & 13 & 16 & 11 & 8 & 14 \\
\hline
\end{tabular}


EDUC4. 4-level education

\begin{tabular}{|l|c|c|c|c|c|}
\hline $\begin{array}{l}\text { NORC } \\
\text { 05/29-06/09/2020 }\end{array}$ & Total & White NH & $\begin{array}{c}\text { African } \\
\text { American } \\
\mathrm{NH}\end{array}$ & Hispanic & Other NH \\
\hline No high school diploma & 16 & 7 & 15 & 35 & 9 \\
\hline $\begin{array}{l}\text { High school graduate or } \\
\text { equivalent }\end{array}$ & 31 & 32 & 35 & 29 & 16 \\
\hline Some college & 25 & 26 & 28 & 18 & 34 \\
\hline $\begin{array}{l}\text { Bachelor's degree or } \\
\text { above }\end{array}$ & 28 & 35 & 22 & 17 & 41 \\
\hline
\end{tabular}

MARITAL. Marital status

\begin{tabular}{|l|c|c|c|c|c|}
\hline $\begin{array}{l}\text { NORC } \\
\text { 05/29-06/09/2020 }\end{array}$ & Total & White NH & $\begin{array}{c}\text { African } \\
\text { American } \\
\mathrm{NH}\end{array}$ & Hispanic & Other NH \\
\hline Married & 53 & 63 & 35 & 48 & 51 \\
\hline Widowed & 8 & 5 & 10 & 9 & 19 \\
\hline Divorced & 15 & 13 & 18 & 15 & 13 \\
\hline Separated & 11 & 10 & 11 & 16 & 6 \\
\hline Never married & 11 & 8 & 21 & 8 & 7 \\
\hline Living with partner & 3 & 1 & 4 & 4 & 5 \\
\hline
\end{tabular}

EMPLOY. Current employment status

\begin{tabular}{|l|c|c|c|c|c|}
\hline $\begin{array}{l}\text { NORC } \\
\text { 05/29-06/09/2020 }\end{array}$ & Total & White NH & $\begin{array}{c}\text { African } \\
\text { American } \\
\text { NH }\end{array}$ & Hispanic & Other NH \\
\hline Working (NET) & $\mathbf{4 1}$ & $\mathbf{4 1}$ & $\mathbf{4 1}$ & $\mathbf{4 0}$ & $\mathbf{3 8}$ \\
\hline $\begin{array}{l}\text { Working - as a paid } \\
\text { employee }\end{array}$ & 34 & 32 & 36 & 35 & 31 \\
\hline Working - self-employed & 7 & 9 & 5 & 5 & 7 \\
\hline Not working (NET) & $\mathbf{6 0}$ & $\mathbf{5 9}$ & $\mathbf{5 9}$ & $\mathbf{6 0}$ & $\mathbf{6 2}$ \\
\hline $\begin{array}{l}\text { Not working - on temporary } \\
\text { layoff from a job }\end{array}$ & 1 & 1 & 2 & 1 & - \\
\hline $\begin{array}{l}\text { Not working - looking for } \\
\text { work }\end{array}$ & 3 & 1 & 1 & 8 & - \\
\hline Not working - retired & 37 & 45 & 32 & 25 & 50 \\
\hline Not working - disabled & 14 & 9 & 22 & 20 & 6 \\
\hline Not working - other & 4 & 4 & 3 & 7 & 6 \\
\hline
\end{tabular}


AARP 50+ Coronavirus Tracker: Wave 5 Oversample

INCOME. Household income

\begin{tabular}{|l|c|c|c|c|c|}
\hline $\begin{array}{l}\text { NORC } \\
05 / 29-06 / 09 / 2020\end{array}$ & Total & White $\mathrm{NH}$ & $\begin{array}{c}\text { African } \\
\text { American } \\
\text { NH }\end{array}$ & Hispanic & Other NH \\
\hline Less than $\$ 50,000(\mathbf{N E T})$ & $\mathbf{4 9}$ & $\mathbf{3 8}$ & $\mathbf{6 4}$ & $\mathbf{5 7}$ & $\mathbf{5 7}$ \\
\hline Less than $\$ 5,000$ & 2 & 1 & 5 & 2 & 5 \\
\hline$\$ 5,000$ to $\$ 9,999$ & 5 & 1 & 10 & 6 & 9 \\
\hline$\$ 10,000$ to $\$ 14,999$ & 6 & 4 & 7 & 8 & - \\
\hline$\$ 15,000$ to $\$ 19,999$ & 5 & 4 & 7 & 5 & 5 \\
\hline$\$ 20,000$ to $\$ 24,999$ & 6 & 5 & 8 & 4 & 8 \\
\hline$\$ 25,000$ to $\$ 29,999$ & 6 & 4 & 5 & 10 & 1 \\
\hline$\$ 30,000$ to $\$ 34,999$ & 6 & 4 & 10 & 7 & 1 \\
\hline$\$ 35,000$ to $\$ 39,999$ & 6 & 7 & 5 & 3 & 13 \\
\hline$\$ 40,000$ to $\$ 49,999$ & 9 & 8 & 8 & 12 & 15 \\
\hline$\$ 50,000$ or $\mathbf{m o r e}(\mathbf{N E T})$ & $\mathbf{5 1}$ & $\mathbf{6 2}$ & $\mathbf{3 6}$ & $\mathbf{4 3}$ & $\mathbf{4 3}$ \\
\hline$\$ 50,000$ to $\$ 59,999$ & 8 & 10 & 7 & 7 & - \\
\hline$\$ 60,000$ to $\$ 74,999$ & 11 & 13 & 8 & 10 & 13 \\
\hline$\$ 75,000$ to $\$ 84,999$ & 6 & 5 & 4 & 7 & 9 \\
\hline$\$ 85,000$ to $\$ 99,999$ & 8 & 10 & 6 & 6 & 2 \\
\hline$\$ 100,000$ to $\$ 124,999$ & 8 & 10 & 5 & 5 & 8 \\
\hline$\$ 125,000$ to $\$ 149,999$ & 3 & 5 & 2 & 2 & 4 \\
\hline$\$ 150,000$ to $\$ 174,999$ & 2 & 3 & 1 & 3 & 3 \\
\hline$\$ 175,000$ to $\$ 199,999$ & 2 & 3 & 2 & 1 & - \\
\hline$\$ 200,000$ or $m 0$ re & 3 & 4 & 1 & 2 & 5 \\
\hline & $N=1,798$ & $N=906$ & $N=401$ & $N=444$ & $N=47$ \\
\hline
\end{tabular}

REGION4. Region - 4 level

\begin{tabular}{|l|c|c|c|c|c|}
\hline $\begin{array}{l}\text { NORC } \\
\text { 05/29-06/09/2020 }\end{array}$ & Total & White NH & $\begin{array}{c}\text { African } \\
\text { American } \\
\mathrm{NH}\end{array}$ & Hispanic & Other NH \\
\hline Northeast & 16 & 20 & 12 & 13 & 18 \\
\hline Midwest & 18 & 25 & 14 & 11 & 4 \\
\hline South & 42 & 35 & 66 & 38 & 27 \\
\hline West & 23 & 20 & 8 & 38 & 51 \\
\hline
\end{tabular}


AARP 50+ Coronavirus Tracker: Wave 5 Oversample

REGION9. Region - 9 level

\begin{tabular}{|l|c|c|c|c|c|}
\hline $\begin{array}{l}\text { NORC } \\
\text { 05/29-06/09/2020 }\end{array}$ & Total & White NH & $\begin{array}{c}\text { African } \\
\text { American } \\
\mathrm{NH}\end{array}$ & Hispanic & Other NH \\
\hline New England & 4 & 6 & 3 & 3 & 3 \\
\hline Mid-Atlantic & 12 & 14 & 9 & 10 & 15 \\
\hline East North Central & 13 & 17 & 12 & 10 & 1 \\
\hline West North Central & 5 & 8 & 2 & 2 & 3 \\
\hline South Atlantic & 24 & 20 & 38 & 20 & 7 \\
\hline East South Central & 6 & 5 & 10 & 5 & 14 \\
\hline West South Central & 12 & 10 & 18 & 13 & 6 \\
\hline Mountain & 7 & 7 & 1 & 11 & 15 \\
\hline Pacific & 16 & 13 & 7 & 27 & 35 \\
\hline
\end{tabular}

STATE. State of residence

\begin{tabular}{|l|c|c|c|c|c|}
\hline $\begin{array}{l}\text { NORC } \\
\text { 05/29-06/09/2020 }\end{array}$ & Total & White NH & $\begin{array}{c}\text { African } \\
\text { American }\end{array}$ & Hispanic & Other NH \\
\hline AK Alaska & $*$ & $*$ & - & - & - \\
\hline AL Alabama & 1 & 1 & 3 & 1 & 4 \\
\hline AR Arkansas & 1 & 2 & $*$ & 1 & 3 \\
\hline AZ Arizona & 2 & 2 & $*$ & 3 & 2 \\
\hline CA California & 12 & 7 & 5 & 26 & 21 \\
\hline CO Colorado & 2 & 3 & $*$ & 2 & 8 \\
\hline CT Connecticut & 1 & 2 & 2 & $*$ & 1 \\
\hline DC District of Columbia & $*$ & $*$ & 2 & - & - \\
\hline DE Delaware & $*$ & $*$ & 1 & $*$ & - \\
\hline FL Florida & 10 & 7 & 8 & 16 & 5 \\
\hline GA Georgia & 3 & 3 & 8 & $*$ & - \\
\hline HI Hawaii & $*$ & $*$ & - & $*$ & 6 \\
\hline IA lowa & 1 & 2 & - & $*$ & - \\
\hline ID Idaho & 1 & 1 & - & 1 & - \\
\hline IL Illinois & 3 & 4 & 4 & 2 & - \\
\hline IN Indiana & 3 & 3 & 3 & 2 & 1 \\
\hline KS Kansas & 1 & 1 & $*$ & $*$ & - \\
\hline KY Kentucky & 1 & 1 & 1 & 2 & - \\
\hline LA Louisiana & 2 & 1 & 5 & 1 & 1 \\
\hline MA Massachusetts & 1 & 2 & $*$ & 1 & - \\
\hline MD Maryland & 2 & 1 & 4 & 1 & - \\
\hline ME Maine & 2 & 1 & - & 1 & - \\
\hline MI Michigan & 2 & 2 & 2 & 1 & - \\
\hline MN Minnesota & & 2 & 2 & 1 & 2 \\
\hline MO Missouri & 2 & & & \\
\hline
\end{tabular}


AARP 50+ Coronavirus Tracker: Wave 5 Oversample

\begin{tabular}{|c|c|c|c|c|c|}
\hline MS Mississippi & 1 & $*$ & 3 & 1 & - \\
\hline MT Montana & $*$ & $*$ & - & $*$ & 2 \\
\hline NC North Carolina & 3 & 3 & 5 & 1 & 1 \\
\hline ND North Dakota & $*$ & $*$ & - & - & - \\
\hline NE Nebraska & 1 & 1 & - & - & - \\
\hline NH New Hampshire & 1 & 1 & - & 1 & - \\
\hline NJ New Jersey & 4 & 4 & 2 & 4 & 6 \\
\hline NM New Mexico & 1 & $*$ & - & 4 & - \\
\hline NV Nevada & 1 & 1 & $*$ & 1 & 2 \\
\hline NY New York & 5 & 4 & 5 & 5 & 5 \\
\hline $\mathrm{OH}$ Ohio & 3 & 3 & 2 & 4 & - \\
\hline OK Oklahoma & $*$ & 1 & $*$ & - & - \\
\hline OR Oregon & 1 & 2 & $*$ & $*$ & 6 \\
\hline PA Pennsylvania & 4 & 6 & 3 & 1 & 4 \\
\hline RI Rhode Island & $*$ & $*$ & $*$ & - & - \\
\hline SC South Carolina & 2 & 2 & 6 & $*$ & - \\
\hline SD South Dakota & $*$ & $*$ & - & - & - \\
\hline TN Tennessee & 3 & 3 & 4 & 1 & 11 \\
\hline TX Texas & 9 & 7 & 12 & 12 & - \\
\hline UT Utah & $*$ & $*$ & - & $*$ & 2 \\
\hline VA Virginia & 3 & 3 & 5 & 2 & 2 \\
\hline VT Vermont & $*$ & $*$ & - & - & 2 \\
\hline WA Washington & 2 & 3 & 1 & 1 & 3 \\
\hline WI Wisconsin & 2 & 3 & 1 & 1 & - \\
\hline WV West Virginia & $*$ & 1 & - & $*$ & - \\
\hline WY Wyoming & $*$ & $*$ & - & $*$ & - \\
\hline
\end{tabular}

\section{METRO. Metropolitan area flag}

\begin{tabular}{|l|c|c|c|c|c|}
\hline $\begin{array}{l}\text { NORC } \\
\text { 05/29-06/09/2020 }\end{array}$ & Total & White NH & $\begin{array}{c}\text { African } \\
\text { American } \\
\mathrm{NH}\end{array}$ & Hispanic & Other NH \\
\hline Non-metro area & 17 & 19 & 12 & 14 & 21 \\
\hline Metro area & 84 & 81 & 88 & 86 & 79 \\
\hline
\end{tabular}

INTERNET. Household internet access

\begin{tabular}{|l|c|c|c|c|c|}
\hline $\begin{array}{l}\text { NORC } \\
\text { 05/29-06/09/2020 }\end{array}$ & Total & White NH & $\begin{array}{c}\text { African } \\
\text { American } \\
\mathrm{NH}\end{array}$ & Hispanic & Other NH \\
\hline Non-internet household & 18 & 9 & 31 & 24 & 22 \\
\hline Internet household & 82 & 91 & 69 & 76 & 78 \\
\hline
\end{tabular}


HOUSING. Home ownership

\begin{tabular}{|l|c|c|c|c|c|}
\hline $\begin{array}{l}\text { NORC } \\
\text { 05/29-06/09/2020 }\end{array}$ & Total & White NH & $\begin{array}{c}\text { African } \\
\text { American } \\
\text { NH }\end{array}$ & Hispanic & Other NH \\
\hline $\begin{array}{l}\text { Owned or being bought by } \\
\text { you or someone in your } \\
\text { household }\end{array}$ & 71 & 82 & 56 & 62 & 83 \\
\hline Rented for cash & 27 & 17 & 43 & 34 & 14 \\
\hline $\begin{array}{l}\text { Occupied without payment of } \\
\text { cash rent }\end{array}$ & 2 & 2 & 1 & 5 & 4 \\
\hline
\end{tabular}

HOME_TYPE. Type of building of panelists' residence

\begin{tabular}{|l|c|c|c|c|c|}
\hline $\begin{array}{l}\text { NORC } \\
\text { 05/29-06/09/2020 }\end{array}$ & Total & White NH & $\begin{array}{c}\text { African } \\
\text { American } \\
\mathrm{NH}\end{array}$ & Hispanic & Other NH \\
\hline $\begin{array}{l}\text { A one-family house detached } \\
\text { from any other house }\end{array}$ & 64 & 73 & 52 & 56 & 63 \\
\hline $\begin{array}{l}\text { A one-family house attached to } \\
\text { one or more houses }\end{array}$ & 9 & 8 & 13 & 6 & 11 \\
\hline $\begin{array}{l}\text { A building with 2 or more } \\
\text { apartments }\end{array}$ & 21 & 14 & 29 & 30 & 13 \\
\hline A mobile home or trailer & 6 & 5 & 7 & 8 & 13 \\
\hline Boat, RV, van, etc. & $*$ & $*$ & - & $*$ & - \\
\hline
\end{tabular}

PHONESERVICE. Telephone service for the household

\begin{tabular}{|l|c|c|c|c|c|}
\hline $\begin{array}{l}\text { NORC } \\
\text { 05/29-06/09/2020 }\end{array}$ & Total & White NH & $\begin{array}{c}\text { African } \\
\text { American } \\
\mathrm{NH}\end{array}$ & Hispanic & Other NH \\
\hline Landline telephone only & 9 & 8 & 10 & 10 & 19 \\
\hline $\begin{array}{l}\text { Have a landline, but mostly } \\
\text { use cellphone }\end{array}$ & 29 & 30 & 34 & 26 & 25 \\
\hline $\begin{array}{l}\text { Have a cellphone, but mostly } \\
\text { use landline }\end{array}$ & 18 & 23 & 16 & 9 & 11 \\
\hline Cellphone only & 43 & 39 & 39 & 56 & 45 \\
\hline No telephone service & $*$ & $*$ & $*$ & $*$ & - \\
\hline
\end{tabular}


AARP 50+ Coronavirus Tracker: Wave 5 Oversample

HHSIZE. Household size (including children)

\begin{tabular}{|l|c|c|c|c|c|}
\hline NORC & Total & White NH & $\begin{array}{c}\text { African } \\
\text { American NH }\end{array}$ & Hispanic & Other NH \\
\hline 1 & 27 & 24 & 33 & 23 & 36 \\
\hline 2 & 43 & 49 & 36 & 36 & 38 \\
\hline 3 & 14 & 14 & 15 & 14 & 8 \\
\hline 4 & 8 & 6 & 8 & 11 & 8 \\
\hline 5 & 4 & 4 & 3 & 7 & 8 \\
\hline $6+$ & 5 & 3 & 4 & 9 & 2 \\
\hline
\end{tabular}

HH01. Number of HH members age 0-1

\begin{tabular}{|l|c|c|c|c|c|}
\hline NORC & Total & White NH & $\begin{array}{c}\text { African } \\
\text { American NH }\end{array}$ & Hispanic & Other NH \\
\hline $05 / 29-06 / 09 / 2020$ & 100 & 100 & 100 & 100 & 100 \\
\hline 0 & $*$ & $*$ & $*$ & $*$ & - \\
\hline 2 & - & - & - & - & - \\
\hline 3 & - & - & - & - & - \\
\hline
\end{tabular}

HH25. Number of HH members age 2-5

\begin{tabular}{|l|c|c|c|c|c|}
\hline $\begin{array}{l}\text { NORC } \\
05 / 29-06 / 09 / 2020\end{array}$ & Total & White NH & $\begin{array}{c}\text { African } \\
\text { American NH }\end{array}$ & Hispanic & Other NH \\
\hline 0 & 98 & 99 & 98 & 95 & 100 \\
\hline 1 & 1 & $*$ & 2 & 3 & - \\
\hline 2 & 1 & $*$ & 1 & 2 & 1 \\
\hline 3 & $*$ & - & - & $*$ & - \\
\hline 4 & - & - & - & - & - \\
\hline 5 & - & - & - & - & - \\
\hline
\end{tabular}


AARP 50+ Coronavirus Tracker: Wave 5 Oversample

HH612. Number of HH members age 6-12

\begin{tabular}{|l|c|c|c|c|c|}
\hline $\begin{array}{l}\text { NORC } \\
05 / 29-06 / 09 / 2020\end{array}$ & Total & White NH & $\begin{array}{c}\text { African } \\
\text { American NH }\end{array}$ & Hispanic & Other NH \\
\hline 0 & 93 & 95 & 92 & 90 & 96 \\
\hline 1 & 5 & 4 & 7 & 5 & 3 \\
\hline 2 & 2 & 1 & 1 & 5 & - \\
\hline 3 & $*$ & $*$ & - & $*$ & - \\
\hline 4 & $*$ & - & $*$ & - & 2 \\
\hline 5 & - & - & - & - & - \\
\hline 6 & $*$ & - & $*$ & - & - \\
\hline 7 & - & - & - & - & - \\
\hline 8 & - & - & - & - & - \\
\hline 9 & - & - & - & - & - \\
\hline 10 & - & - & - & - & - \\
\hline
\end{tabular}

HH1317. Number of HH members age 13-17

\begin{tabular}{|l|c|c|c|c|c|}
\hline NORC & Total & White NH & $\begin{array}{c}\text { African } \\
\text { American NH }\end{array}$ & Hispanic & Other NH \\
\hline 0 & 93 & 95 & 95 & 88 & 95 \\
\hline 1 & 5 & 4 & 5 & 7 & 3 \\
\hline 2 & 2 & 1 & 1 & 4 & 2 \\
\hline 3 & $*$ & $*$ & $*$ & 1 & - \\
\hline 4 & $*$ & - & - & 1 & - \\
\hline 5 & - & - & - & - & - \\
\hline 6 & - & - & - & - & - \\
\hline 7 & - & - & - & - & - \\
\hline
\end{tabular}


AARP 50+ Coronavirus Tracker: Wave 5 Oversample

HH18OV. Number of HH members age 18+

\begin{tabular}{|l|c|c|c|c|c|}
\hline NORC & Total & White NH & $\begin{array}{c}\text { African } \\
\text { American NH }\end{array}$ & Hispanic & Other NH \\
\hline 1 & 34 & 30 & 41 & 34 & 46 \\
\hline 2 & 43 & 50 & 40 & 34 & 33 \\
\hline 3 & 13 & 12 & 11 & 17 & 10 \\
\hline 4 & 6 & 5 & 6 & 9 & 10 \\
\hline 5 & 2 & 2 & 1 & 4 & - \\
\hline 6 & 1 & 1 & 1 & 1 & - \\
\hline 7 & $*$ & $*$ & $*$ & $*$ & - \\
\hline 8 & $*$ & $*$ & $*$ & $*$ & - \\
\hline 9 & $*$ & - & - & $*$ & - \\
\hline 10 & $*$ & $*$ & - & $*$ & - \\
\hline
\end{tabular}

PANEL_TYPE PRELOAD. Sample source/type

\begin{tabular}{|l|c|c|c|c|c|}
\hline $\begin{array}{l}\text { NORC } \\
\text { 05/29-06/09/2020 }\end{array}$ & Total & White NH & $\begin{array}{c}\text { African } \\
\text { American } \\
\mathrm{NH}\end{array}$ & Hispanic & Other $\mathrm{NH}$ \\
\hline AmeriSpeak & 62 & 47 & 70 & 85 & 68 \\
\hline UTellUS & 38 & 53 & 30 & 15 & 32 \\
\hline
\end{tabular}

P_LGBT PRELOAD.

\begin{tabular}{|l|c|c|c|c|c|}
\hline $\begin{array}{l}\text { NORC } \\
\text { 05/29-06/09/2020 }\end{array}$ & Total & White NH & $\begin{array}{c}\text { African } \\
\text { American NH }\end{array}$ & Hispanic & Other NH \\
\hline Lesbian or gay & 2 & 2 & 2 & 2 & 3 \\
\hline $\begin{array}{l}\text { Straight, that is not lesbian or } \\
\text { gay }\end{array}$ & 95 & 95 & 95 & 95 & 94 \\
\hline Bisexual & 1 & 1 & 2 & $*$ & 4 \\
\hline Something Else & 1 & 1 & 1 & 1 & - \\
\hline I don't know the answer & $*$ & $*$ & $*$ & 1 & - \\
\hline Unknown & $*$ & $*$ & $*$ & 1 & - \\
\hline
\end{tabular}


P_VOTENEW PRELOAD.

\begin{tabular}{|l|c|c|c|c|c|}
\hline NORC & Total & White NH & $\begin{array}{c}\text { African } \\
\text { American NH }\end{array}$ & Hispanic & Other NH \\
\hline Registered at current address & 87 & 91 & 89 & 77 & 91 \\
\hline Registered at a different address & 3 & 3 & 3 & 2 & 1 \\
\hline Not currently registered & 8 & 5 & 7 & 14 & 8 \\
\hline I am not eligible to vote & 2 & 1 & 1 & 6 & - \\
\hline Not Sure & $*$ & $*$ & $*$ & 1 & 1 \\
\hline
\end{tabular}

P_PARTYID7 PRELOAD.

\begin{tabular}{|l|c|c|c|c|c|}
\hline NORC & Total & White NH & $\begin{array}{c}\text { African } \\
\text { American NH }\end{array}$ & Hispanic & Other NH \\
\hline Strong Democrat & 20 & 12 & 42 & 16 & 12 \\
\hline Moderate Democrat & 23 & 17 & 33 & 27 & 19 \\
\hline Lean Democrat & 9 & 10 & 9 & 7 & 20 \\
\hline Don't Lean/Independent/None & 14 & 12 & 12 & 18 & 22 \\
\hline Lean Republican & 8 & 12 & 1 & 7 & 4 \\
\hline Moderate Republican & 15 & 22 & 3 & 11 & 14 \\
\hline Strong Republican & 12 & 15 & 1 & 14 & 9 \\
\hline Unknown & $*$ & - & $*$ & $*$ & - \\
\hline
\end{tabular}

\section{P_IDEO PRELOAD.}

\begin{tabular}{|c|c|c|c|c|c|}
\hline $\begin{array}{l}\text { NORC } \\
05 / 29-06 / 09 / 2020\end{array}$ & Total & White NH & $\begin{array}{c}\text { African } \\
\text { American } \mathrm{NH}\end{array}$ & Hispanic & Other NH \\
\hline Extremely Liberal & 6 & 5 & 7 & 7 & 6 \\
\hline Liberal & 15 & 16 & 18 & 11 & 17 \\
\hline Slightly Liberal & 11 & 12 & 10 & 9 & 6 \\
\hline Moderate & 25 & 22 & 31 & 25 & 31 \\
\hline Slightly Conservative & 7 & 9 & 4 & 7 & 11 \\
\hline Conservative & 20 & 23 & 9 & 23 & 19 \\
\hline Extremely Conservative & 5 & 6 & 2 & 5 & 4 \\
\hline Haven't thought much about it & 11 & 8 & 18 & 13 & 7 \\
\hline Unknown & $*$ & - & $*$ & - & - \\
\hline
\end{tabular}

P_MEMBERAARP PRELOAD.

\begin{tabular}{|l|c|c|c|c|c|}
\hline $\begin{array}{l}\text { NORC } \\
\text { 05/29-06/09/2020 }\end{array}$ & Total & White NH & $\begin{array}{c}\text { African } \\
\text { American NH }\end{array}$ & Hispanic & Other NH \\
\hline Member & 46 & 52 & 52 & 32 & 30 \\
\hline Non-member & 54 & 48 & 48 & 68 & 70 \\
\hline
\end{tabular}




\section{STUDY METHODOLOGY}

This survey was conducted by the AmeriSpeak Panel department of NORC at the University of Chicago. Data were collected using the probability-based, nationally representative AmeriSpeak ${ }^{\circledR}$ Panel.

AmeriSpeak conducts surveys for experts who can't afford to be wrong.

Since its founding by NORC at the University of Chicago in 2015, AmeriSpeak has produced more than 500 surveys, been cited by dozens of media outlets ${ }^{1}$ and become the primary survey partner of the nation's preeminent news service, The Associated Press. AmeriSpeak has the highest AAPOR response rate among commercially available household survey panels in the U.S. AmeriSpeak captures a true picture of America, providing better representation than other survey panels for hard-to-reach populations, including low-income households, less educated persons, young adults, rural households, persons who are less interested in the news, and social and political conservatives.

Technical information about the AmeriSpeak Panel is available here.

During the initial recruitment phase of the AmeriSpeak Panel, randomly selected U.S. households were sampled with a known, non-zero probability of selection from the NORC National Sample Frame, supplemented with households selected from the USPS Delivery Sequence File. Selected U.S. households were then contacted by U.S. mail, email, telephone, and field interviewers (face-to-face interviewing in the homes of respondents). AmeriSpeak provides sample coverage of approximately 97 percent of the U.S. household population. Those excluded from the sample include people in groupliving quarters and those with P.O. Box only addresses and some newly constructed dwellings.

AmeriSpeak Panel members selected for this study were scientifically selected to represent persons age 50 and over in the 50 states and the District of Columbia. There were 1,798 AmeriSpeak respondents completing the survey-1,598 via the web and 200 via telephone. Interviews were conducted in English and Spanish. The final stage survey completion rate is 20.2 percent. The overall margin of sampling error is $+/-3.37$ percentage points at the 95 percent confidence level for a $50 \%$ survey statistic, including the design effect. The margin of sampling error may be higher for subgroups.

Once the AmeriSpeak study sample has been selected and fielded, and all the study data have been collected and made final, a statistical weighting process is used to adjust for any survey nonresponse as well as any noncoverage or under and oversampling resulting from the study specific sample design. Poststratification variables included age, gender, census division, race/ethnicity, and education.

\footnotetext{
${ }^{1}$ AmeriSpeak research has been cited in major media outlets including Time Magazine, The New York Times, CNN, Forbes, Chicago Tribune, and others. NORC's AmeriSpeak clients represent a broad range of federal, foundation, non-profit, and private sector organizations, including not limited to: AARP, U.S. Centers for Disease Control \& Prevention, Consumer Reports, Kaiser Family Foundation, Legal Services Corp. of America, National Science Foundation, NASA, National Cancer Institute, National Institute of Justice, Pew Research Center, The New York Times, Robert Wood Johnson Foundation, Truth Initiative, dozens of university-based researchers, among others. In addition, NORC's AmeriSpeak Panel is the sample source for several studies that have been approved by the United States Office of Management and Budget (OMB), including studies sponsored by the U.S. Centers for Disease Control \& Prevention, the US. Department of Defense, and the Internal Revenue Service.
} 
The same survey nonresponse adjustment process was applied to the overall sample, which included the general population sample and the oversamples. Overall poststratification variables included RACEETH3 (3 categories race/ethnicity: Hispanics, Non-Hispanic African American, and Non-Hispanic Other) * age, RACEETH3 * gender, RACEETH3 * education, census division, and race/ethnicity (4 categories: Hispanics, Non-Hispanic African American, Non-Hispanic White, and Non-Hispanic Other). One population weight summing to the population size was created. Then we normalized the population weight differently to create two normalized weights, which summed to the number of completes. WEIGHT was normalized overall. The proportions of RACEETH3 reflected the proportions in the population. WEIGHT2 was normalized by RACEETH3. The proportions of RACEETH3 reflected the proportions in the completes. WEIGHT should be used when analyzing the general population. WEIGHT2 could be used to analyze specific race/ethnicity subgroups.

Weighting variables were obtained from the 2019 Current Population Survey. The weighted data reflect the U.S. population of adults age 50 and over. For more information, email amerispeak-bd@norc.org.

\section{ABOUT NORC AT THE UNIVERSITY OF CHICAGO}

NORC at the University of Chicago is an objective, non-partisan research institution that delivers reliable data and rigorous analysis to guide critical programmatic, business, and policy decisions. Since 1941, NORC has conducted groundbreaking studies, created and applied innovative methods and tools, and advanced principles of scientific integrity and collaboration. Today, government, corporate, and nonprofit clients around the world partner with NORC to transform increasingly complex information into useful knowledge.

www.norc.org 